PHYSICAL REVIEW D 80, 035018 (2009)

\title{
Rare meson decays into very light neutralinos
}

\author{
H. K. Dreiner* and S. Grab ${ }^{\dagger}$ \\ Bethe Center for Theoretical Physics \& Physikalisches Institut der Universität Bonn, Nußallee 12, 53115 Bonn, Germany \\ Daniel Koschade \\ Institut für Theoretische Physik, RWTH Aachen University, 52056 Aachen, Germany \\ and Centre for Research in String Theory, Department of Physics, Queen Mary, University of London, \\ E1 4NS London, United Kingdom \\ M. Krämer ${ }^{\S}$ and Ben O’Leary"l \\ Institut für Theoretische Physik, RWTH Aachen University, 52056 Aachen, Germany \\ Ulrich Langenfeld ${ }^{\text {II }}$ \\ DESY, Platanenallee 6, D-15738 Zeuthen, Germany
}

(Received 20 May 2009; published 21 August 2009)

\begin{abstract}
We investigate the bounds on the mass of the lightest neutralino from rare meson decays within the minimal supersymmetric standard model (MSSM) with and without minimal flavor violation. We present explicit formulas for the two-body decays of mesons into light neutralinos and perform the first complete calculation of the loop-induced decays of kaons to pions and light neutralinos and $B$ mesons to kaons and light neutralinos. We find that the supersymmetric branching ratios are strongly suppressed within the MSSM with minimal flavor violation, and that no bounds on the neutralino mass can be inferred from experimental data, i.e., a massless neutralino is allowed. The branching ratios for kaon and $B$ meson decays into light neutralinos may, however, be enhanced when one allows for nonminimal flavor violation. We find new constraints on the MSSM parameter space for such scenarios and discuss prospects for future kaon and $B$ meson experiments. Finally, we comment on the search for light neutralinos in monojet signatures at the Tevatron and at the LHC.
\end{abstract}

DOI: 10.1103/PhysRevD.80.035018

PACS numbers: 12.60.Jv, 13.20.Eb, 13.20.Gd, 13.20.He

\section{INTRODUCTION}

Supersymmetry (SUSY) is an attractive candidate for physics beyond the standard model (SM) [1-3]. In the minimal supersymmetric extension of the SM (MSSM) the superpartners of the electroweak gauge and Higgs bosons (the gauginos and Higgsinos) mix to form two electrically charged and four electrically neutral mass eigenstates, called charginos, $\tilde{\chi}_{1,2}^{ \pm}$, and neutralinos, $\tilde{\chi}_{1,2,3,4}^{0}$, respectively. In many supersymmetric models, the neutralino $\tilde{\chi}_{1}^{0}$ is the lightest supersymmetric particle (LSP) and thus plays a special role in phenomenology. In particular, in models with conserved proton hexality [4] (or conserved $R$ parity [5]) a neutralino LSP is stable and provides a promising dark matter candidate [6-8].

So far, no evidence for supersymmetric particles has been found, and lower limits on sparticle masses have been derived from searches at colliders and from precision

\footnotetext{
*dreiner@th.physik.uni-bonn.de

sgrab@th.physik.uni-bonn.de

¥.koschade@qmul.ac.uk

\$mkraemer@physik.rwth-aachen.de

"oleary@physik.rwth-aachen.de

"ulrichl@ifh.de
}

measurements of low-energy observables. The particle data group (PDG) [9] quotes a lower limit on the mass of the lightest neutralino [10]

$$
m_{\tilde{\chi}_{1}^{0}}>46 \mathrm{GeV} \quad(95 \% \text { C.L. }) .
$$

This limit, however, is not model independent but assumes the unification of the gaugino mass parameters $M_{1}$ and $M_{2}$ at some high energy scale. The renormalization group evolution of the mass parameters then implies

$$
M_{1}=\frac{5}{3} \frac{g^{\prime 2}}{g^{2}} M_{2}=\frac{5}{3} \tan ^{2} \theta_{W} M_{2} \approx \frac{1}{2} M_{2}
$$

at the electroweak scale, where $g$ and $g^{\prime}$ denote the $S U(2)_{L}$ and $U(1)_{Y}$ gauge couplings, respectively, and $\theta_{W}$ is the weak mixing angle. The experimental mass bound for the lighter chargino, $m_{\tilde{\chi}_{1}^{ \pm}}>94 \mathrm{GeV}$ (95\% C.L.) [9], places a lower bound on $M_{2}$ (and on the Higgsino mass parameter $\mu$ ) and indirectly, through Eq. (2), on $M_{1}$. The bounds on $M_{1}, M_{2}$, and $\mu$, as well as the lower bound on $\tan \beta \equiv$ $v_{2} / v_{1}$ (the ratio of the two vacuum expectation values of the MSSM Higgs doublets) from the LEP Higgs searches [11], then in turn give rise to the lower bound on the mass of the lightest neutralino, Eq. (1). 
In this paper, we investigate a more general MSSM scenario where the assumption Eq. (2) is dropped and the gaugino mass parameters $M_{1}$ and $M_{2}$ are treated as independent parameters. It has been shown that in such a scenario, one can adjust $M_{1}$ and $M_{2}$ such that the lightest neutralino is massless [12-14]. Specifically, $m_{\tilde{\chi}_{1}^{0}}=0$ if

$$
\begin{aligned}
M_{1} & =\frac{M_{2} m_{Z}^{2} \sin (2 \beta) \sin ^{2} \theta_{W}}{\mu M_{2}-m_{Z}^{2} \sin (2 \beta) \cos \theta_{W}} \approx \frac{m_{Z}^{2} \sin (2 \beta) \sin ^{2} \theta_{W}}{\mu} \\
& \approx 2.5 \mathrm{GeV}\left(\frac{10}{\tan \beta}\right)\left(\frac{150 \mathrm{GeV}}{\mu}\right),
\end{aligned}
$$

where $m_{Z}$ is the $Z$ gauge boson mass. The lower bound on the lightest chargino mass, $m_{\tilde{\chi}_{1}^{ \pm}}>94 \mathrm{GeV}$, leads to lower bounds of $|\mu|, M_{2} \gtrsim 100 \mathrm{GeV}$ [15]. Thus, adjusting $M_{1}$ according to Eq. (3) so that $m_{\tilde{\chi}_{1}^{0}}=0$ implies $M_{1} \ll M_{2}, \mu$. In this region of parameter space, the bino component of the lightest neutralino is above $90 \%$, i.e., the lightest neutralino couples predominantly to hypercharge $[14,16]$.

Note that choosing a renormalization scheme in which $m_{\tilde{\chi}_{1}^{0}}$ is an input parameter $[17,18]$ implies that a small $\tilde{\chi}_{1}^{0}$ mass at tree level will remain small also after radiative corrections. However, note that a small or zero neutralino mass cannot always be obtained in the presence of nonzero complex phases for $M_{1}$ and $\mu$ [18]. (One can always choose a convention where $M_{2}$ is real by absorbing its phase into a redefinition of the gaugino fields.)

The phenomenology of a very light or massless neutralino has been discussed by many previous authors, in particular, with respect to its astrophysical, see e.g., Refs. [14,19-21], and cosmological implications, see e.g., [18,22-26]. Light neutralinos have also been discussed in the context of collider searches [15,27-32], as well as electroweak precision observables $[18,33]$ and rare meson decays [18,34-38]. In a previous paper [18], some of the present authors presented an extensive study of these phenomenological constraints. While a very light or massless neutralino cannot provide the cold dark matter content of the Universe, it is consistent with all existing laboratory constraints and astrophysical and cosmological observations. For a comprehensive review of the literature on very light neutralinos, we refer to Ref. [18].

In the present work we focus on a specific aspect of the phenomenology of light neutralinos, namely, rare meson decays to light neutralinos. For more than three decades, rare meson decays have been considered a sensitive test for the presence of new physics. In this paper, we investigate the tree-level decay of pseudoscalar and vector mesons and the loop-induced decay of kaons to pions and light neutralinos and $B$ mesons to kaons and light neutralinos within the MSSM. A review and an update of the literature has been presented in a recent accompanying paper, Ref. [18]. We go beyond Ref. [18] and the previous work in several aspects. First, we provide details of the calculations and explicit formulas for the two-body decays of mesons $M$ into light neutralinos, $M \rightarrow \tilde{\chi}_{1}^{0} \tilde{\chi}_{1}^{0}$, that will allow one to estimate the decay width $\Gamma\left(M \rightarrow \tilde{\chi}_{1}^{0} \tilde{\chi}_{1}^{0}\right)$ also in generic supersymmetric theories beyond the MSSM. Moreover, we perform the first complete calculation of the loop-induced decays $K^{-} \rightarrow \pi^{-} \tilde{\chi}_{1}^{0} \tilde{\chi}_{1}^{0}$ and $B^{-} \rightarrow K^{-} \tilde{\chi}_{1}^{0} \tilde{\chi}_{1}^{0}$ in the MSSM with minimal flavor violation (MFV) [39]. We show that these decays are strongly suppressed with respect to the SM decays $K^{-} \rightarrow \pi^{-} \nu \bar{\nu}$ and $B^{-} \rightarrow K^{-} \nu \bar{\nu}$. We also consider nonminimal flavor violation in the MSSM and demonstrate that rare kaon and $B$ meson decays do provide new constraints on the supersymmetric parameter space in the case of light or massless neutralinos. Finally, we comment on the direct search for light neutralinos in monojet signatures at the Tevatron and at the LHC.

We note that this MSSM with extremely light neutralinos is not the only model which allows for invisible meson decays into beyond-the-SM (BSM) particles. Other models can have similar decays, which may be within reach of near-future experiments. The standard model can be simply extended to accommodate light gauge singlet scalars which are dark matter candidates [40-42], though it requires some fine-tuning. Rare meson decays to the scalars in this model have been calculated in Refs. [43,44]. These decays in more complex, less fine-tuned models [45,46], in which the light scalars couple to new heavy fermions or a new light $U(1)^{\prime}$ gauge boson, have been calculated in Refs. [47-49]. Models with extra gauged $U(1)$ symmetries also allow for light fermionic dark matter candidates [50,51], and the rare meson decays into these fermions have been calculated in Refs. [48,49]. The next-to-minimal supersymmetric standard model (NMSSM) also allows for light neutralinos, and in the NMSSM they remain a dark matter candidate [25]; the NMSSM also admits a light pseudoscalar dark matter candidate. The rare meson decays into invisible BSM particles in the NMSSM have been calculated in Refs. [44,52]. Another supersymmetric model is that of Ref. [53], a model with gauge mediation of supersymmetry breaking with hidden sectors, and in these hidden sectors reside candidates for light dark matter. Decays of mesons into these dark matter candidates have been calculated in Ref. [54].

The paper is structured as follows. In Sec. II we present the calculation of the decay of pseudoscalar and vector mesons to light neutralinos and compare the results with existing experimental bounds. Since the decay amplitudes involve virtual squarks which, in the MSSM, are generically heavy, $m_{\tilde{q}} \geqslant 100 \mathrm{GeV}$, the branching ratios to neutralinos are small, well below the experimental upper bounds. We provide explicit formulas which allow one to study the dependence of the decay rate on the generic supersymmetric masses and mixings. In Sec. III we discuss the complete calculation of the loop-induced decays $\mathrm{K}^{-} \rightarrow$ $\pi^{-} \tilde{\chi}_{1}^{0} \tilde{\chi}_{1}^{0}$ and $B^{-} \rightarrow K^{-} \tilde{\chi}_{1}^{0} \tilde{\chi}_{1}^{0}$ in the MSSM with minimal flavor violation. We present numerical results for various 
supersymmetric scenarios and find that the branching ratios are several orders of magnitude smaller than the SM processes with neutrinos instead of neutralinos in the final state. The branching ratios for the $K^{-} \rightarrow \pi^{-} \tilde{\chi}_{1}^{0} \tilde{\chi}_{1}^{0}$ and $B^{-} \rightarrow K^{-} \tilde{\chi}_{1}^{0} \tilde{\chi}_{1}^{0}$ decays may, however, be greatly enhanced when one allows for nonminimal flavor violation in the MSSM, as discussed in Sec. IV. We find new constraints on the MSSM parameter space and argue that future experiments in the kaon and $B$ meson sector may be able to probe light neutralinos with masses up to approximately $1 \mathrm{GeV}$ through rare decays. We comment on direct searches for light neutralinos with a monojet signature at hadron colliders in Sec. V and conclude in Sec. VI. The Appendix provides details on the form factors needed for the calculation of the kaon and $B$ meson decays.

\section{MESON DECAYS INTO LIGHT NEUTRALINOS}

In this section, we consider the decays of the electrically neutral, flavorless mesons $M=\pi^{0}, \eta, \eta^{\prime}, \rho^{0}, \omega, \phi, J / \psi$, and $Y$ into a pair of light binolike neutralinos, $M \rightarrow \tilde{\chi}_{1}^{0} \tilde{\chi}_{1}^{0}$. We are thus considering the decay of an invisible particle into other invisible particles, analogous to meson decays into neutrinos in the SM. Such decays have been studied and searched for experimentally, making clever use of kinematics in decay chains. For example, an invisibly decaying $\pi^{0}$ can be reconstructed from a $K^{+} \rightarrow \pi^{+} \pi^{0}$ decay [55], while an invisibly decaying $J / \psi$ may be observed by tagging on the invariant mass of the di-pion system in the decay $\psi(2 S) \rightarrow J / \psi+\pi \pi$ [56]. The decays of pseudoscalar and vector mesons to light photinolike neutralinos have first been studied in Refs. [3,34,35,38,57,58]. More recent calculations addressing $B$ meson and $Y$ decays into light new matter in the MSSM and beyond can be found in Refs. [47,59-61]. We comment on the comparison with existing results when discussing the individual decays below.

Note that while decays with an additional photon in the final state such as $Y \rightarrow \tilde{\chi}_{1}^{0} \tilde{\chi}_{1}^{0}+\gamma$ are experimentally easier to detect, they suffer from a further suppression by a factor of $\alpha$. Since the branching ratios we obtain for the two-body decays $M \rightarrow \tilde{\chi}_{1}^{0} \tilde{\chi}_{1}^{0}$ are extremely small anyway, we do not pursue the radiative decays $M \rightarrow \tilde{\chi}_{1}^{0} \tilde{\chi}_{1}^{0}+\gamma$.

The tree-level contributions to the decays of pseudoscalar $(P)$ or vector $(V)$ mesons, $P / V \rightarrow \tilde{\chi}_{1}^{0} \tilde{\chi}_{1}^{0}$, are mediated
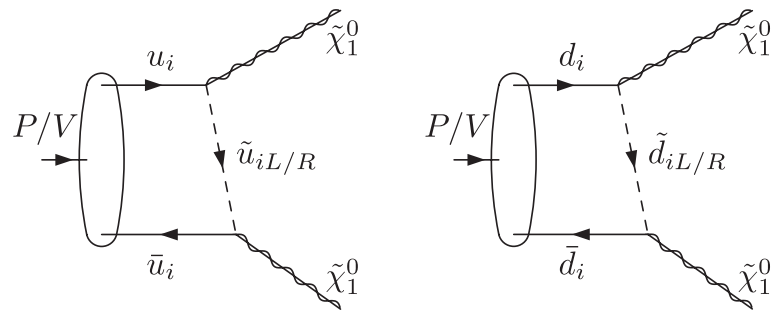

FIG. 1. Feynman diagrams for the decay $P / V \rightarrow \tilde{\chi}_{1}^{0} \tilde{\chi}_{1}^{0}$, omitting diagrams with the $\tilde{\chi}_{1}^{0}$ 's crossed. by $\tilde{u}_{i L / R}$ and $\tilde{d}_{i L / R} t$-channel exchange, as shown in Fig. 1. Here $\tilde{u}_{i L / R} / \tilde{d}_{i L / R}$ denote a left- or right-handed up or down squark of flavor $i$.

\section{A. Decays of pseudoscalar mesons}

We first consider the case $\pi^{0} \rightarrow \tilde{\chi}_{1}^{0} \tilde{\chi}_{1}^{0}$, and then generalize to the other pseudoscalar meson decays. Since the branching ratio depends on the ratio $m_{\tilde{\chi}_{1}^{0}} / m_{P}$ in exactly the same way for each pseudoscalar meson $P$ and differs only by an overall factor, we plot the dependence once, normalized to the peak branching ratio, in Fig. 2. Throughout this paper, to avoid unnecessary clutter, we remove the superscripts denoting charges for mesons in subscripts, e.g., we write $m_{\pi}$ for the mass of the neutral pion, rather than $m_{\pi^{0}}$, and we ignore the differences in masses between charged and uncharged mesons, so $m_{\pi}$ is also used for the mass of the charged pion.

The decay of a pseudoscalar meson into a fermionantifermion pair requires at least one of the fermions to be massive, due to the required helicity flip. In Fig. 2 we see that the branching ratio goes to zero for vanishing neutralino mass. Hence pseudoscalar decays offer no bounds for models with massless neutralinos. Instead, the peak branching ratio is reached for $m_{\tilde{\chi}_{1}^{0}} / m_{P}=1 / \sqrt{6} \approx$ 0.41 .

Likewise, the SM background of $P \rightarrow \nu_{i} \bar{\nu}_{i}, i=e, \mu$, and $\tau$ is extremely small. Its branching ratio is proportional to the neutrino mass squared divided by the pion mass squared. Even for the pion and relatively heavy neutrinos with $m_{\nu_{i}}=1 \mathrm{eV}$, this is of the order of $10^{-10}$.

$$
\text { 1. } \pi^{0} \rightarrow \tilde{\chi}_{1}^{0} \tilde{\chi}_{1}^{0}
$$

We treat the neutral pion as a bound state of valence quarks, given in terms of quark field bilinears as $(u \bar{u}-$ $d \bar{d}) / \sqrt{2}$. Denoting the neutralino momenta by $k_{1}$ and $k_{2}$, the matrix element for this decay is given by

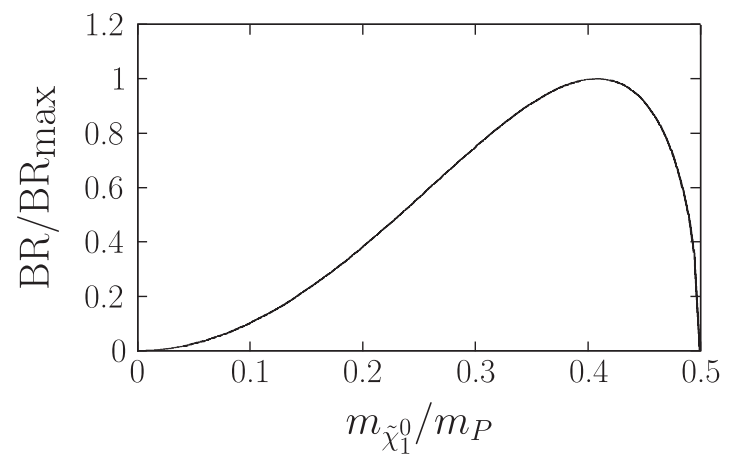

FIG. 2. The branching ratio for a pseudoscalar meson decay $P \rightarrow \tilde{\chi}_{1}^{0} \tilde{\chi}_{1}^{0}$ against the ratio $m_{\tilde{\chi}_{1}^{0}} / m_{P}$ normalized to the peak branching ratio. 


$$
\begin{aligned}
\mathcal{M}= & \frac{1}{i}\left\langle\tilde{\chi}_{1}^{0} \tilde{\chi}_{1}^{0}\right|\left[\overline{\tilde{\chi}}_{1}^{0}\left(i|e| a_{u} \tilde{u}_{L}^{*} P_{L}+i|e| b_{u} \tilde{u}_{R}^{*} P_{R}\right) u+\overline{\tilde{\chi}}_{1}^{0}\left(i|e| a_{d} \tilde{d}_{L}^{*} P_{L}+i|e| b_{d} \tilde{d}_{R}^{*} P_{R}\right) d\right] \\
& \times\left[\bar{u}\left(-i|e| a_{u} \tilde{u}_{L} P_{R}-i|e| b_{u} \tilde{u}_{R} P_{L}\right) \tilde{\chi}_{1}^{0}+\bar{d}\left(-i|e| a_{d} \tilde{d}_{L} P_{R}-|e| i b_{d} \tilde{d}_{R} P_{L}\right) \tilde{\chi}_{1}^{0}\right]\left|\pi^{0}\right\rangle \\
= & \frac{e^{2}}{8} \bar{u}_{\tilde{\chi}_{1}^{0}}\left(k_{1}\right) \gamma_{\mu} \gamma^{5} v_{\tilde{\chi}_{1}^{0}}\left(k_{2}\right)\left\langle 0\left|\left(\frac{a_{u}^{2}}{m_{\tilde{u}_{L}}^{2}}+\frac{b_{u}^{2}}{m_{\tilde{u}_{R}}^{2}}\right) \bar{u} \gamma^{\mu} \gamma^{5} u+\left(\frac{a_{d}^{2}}{m_{\tilde{d}_{L}}^{2}}+\frac{b_{d}^{2}}{m_{\tilde{d}_{R}}^{2}}\right) \bar{d} \gamma^{\mu} \gamma^{5} d\right| \pi^{0}\right\rangle-\left(k_{1} \leftrightarrow k_{2}\right) .
\end{aligned}
$$

In the last line $\bar{u}_{\tilde{\chi}_{1}^{0}}\left(k_{1}\right)$ and $v_{\tilde{\chi}_{1}^{0}}\left(k_{2}\right)$ denote the standard 4component Dirac wave functions (for the neutralinos), whereas $u, \bar{u}, d$, and $\bar{d}$ refer to the corresponding quarks. We have approximated the squark propagators by $-i / m_{\tilde{q}_{L / R}}^{2}$, since the pion mass $m_{\pi}$ (and in general all the pseudoscalar masses $\left.m_{P}\right) \ll m_{\tilde{q}}$. In order to obtain the last line we made use of the fact that the pion is a pseudoscalar state, i.e., $\left\langle 0\left|\bar{u} \gamma^{\mu} u\right| \pi^{0}\right\rangle=\left\langle 0\left|\bar{d} \gamma^{\mu} d\right| \pi^{0}\right\rangle=0$. Since the neutralino is a Majorana fermion, we also have $\overline{\tilde{\chi}}_{1}^{0} \gamma^{\mu} \tilde{\chi}_{1}^{0}=$ $\overline{\tilde{\chi}}_{1 L}^{0} \sigma^{\mu} \tilde{\chi}_{1 L}^{0}+\tilde{\chi}_{1 L}^{0} \bar{\sigma}^{\mu} \overline{\tilde{\chi}}_{1 L}^{0}=0$ identically. We used Fierz identities $[62,63]$ to transform the products of spinor bilinears to the presented form.

For purely bino neutralinos, the neutralino-quark-squark couplings are $|e| a_{q} P_{L}$ for an outgoing $\tilde{q}_{L}$ and $|e| b_{q} P_{R}$ for an outgoing $\tilde{q}_{R}$, and since the gauge couplings are generation independent, we just write $a_{u}$ for up-type quarks and $a_{d}$ for down-type quarks, with

$$
\begin{gathered}
a_{u}=a_{d}=\frac{-1}{3 \sqrt{2} \cos \theta_{W}}, \quad b_{u}=\frac{2 \sqrt{2}}{3 \cos \theta_{W}}, \\
b_{d}=\frac{-\sqrt{2}}{3 \cos \theta_{W}} .
\end{gathered}
$$

$e$ is the electromagnetic charge of the electron, such that $e^{2}=4 \pi \alpha$. We take $\alpha=1 / 137$ and the electroweak mixing angle $\sin ^{2} \theta_{W}=0.23$. Equation (4) can be seen to agree with Eqs. (2) and (3) in Ref. [38], if the neutralino-quarksquark couplings are chosen to be photinolike instead of binolike (i.e., $a_{u}=b_{u}=2 \sqrt{2} / 3$ and $a_{d}=b_{d}=-\sqrt{2} / 3$ ) and the squark mixing $\mu^{2}$ therein is set to zero.

We use the standard definition of the pseudoscalar decay constant $[64,65]$ to write

$$
\left\langle 0\left|\bar{u} \gamma^{\mu} \gamma^{5} u\right| \pi^{0}\right\rangle=-\left\langle 0\left|\bar{d} \gamma^{\mu} \gamma^{5} d\right| \pi^{0}\right\rangle=\frac{i}{\sqrt{2}} F_{\pi} p_{\pi}^{\mu},
$$

where $p_{\pi}$ is the momentum of the pion. Equation (6) defines our convention for the value of the pion decay constant $F_{\pi}$ as $131 \mathrm{MeV}$ [9] (as opposed to the other common convention of $91 \mathrm{MeV}$, which incorporates the factor of $1 / \sqrt{2}$ ).

Summing over final spins, the square of the matrix element is given by

$$
\begin{aligned}
\overline{|\mathcal{M}|^{2}}= & \frac{e^{4} F_{\pi}^{2} m_{\tilde{\chi}_{1}^{0}}^{2}}{8 m_{\pi}^{2}}\left(2 p_{\pi} \cdot k_{1} p_{\pi} \cdot k_{2}-m_{\pi}^{2} k_{1} \cdot k_{2}\right. \\
& \left.+m_{\pi}^{2} m_{\tilde{\chi}_{1}^{0}}^{2}\right)\left(\frac{a_{u}^{2}}{m_{\tilde{u}_{L}}^{2}}+\frac{b_{u}^{2}}{m_{\tilde{u}_{R}}^{2}}-\frac{a_{d}^{2}}{m_{\tilde{d}_{L}}^{2}}-\frac{b_{d}^{2}}{m_{\tilde{d}_{R}}^{2}}\right)^{2},
\end{aligned}
$$

leading to a partial decay width of

$$
\begin{aligned}
\Gamma_{\pi^{0} \rightarrow \tilde{\chi}_{1}^{0} \tilde{\chi}_{1}^{0}}= & \frac{\alpha^{2} \pi}{8} F_{\pi}^{2} m_{\tilde{\chi}_{1}^{0}}^{2} m_{\pi} \sqrt{1-4\left(\frac{m_{\tilde{\chi}_{1}^{0}}}{m_{\pi}}\right)^{2}} \\
& \times\left(\frac{a_{u}^{2}}{m_{\tilde{u}_{L}}^{2}}+\frac{b_{u}^{2}}{m_{\tilde{u}_{R}}^{2}}-\frac{a_{d}^{2}}{m_{\tilde{d}_{L}}^{2}}-\frac{b_{d}^{2}}{m_{\tilde{d}_{R}}^{2}}\right)^{2} .
\end{aligned}
$$

If we assume a common squark mass $m_{\tilde{q}}$, then we obtain a branching ratio of

$$
\begin{aligned}
\operatorname{BR}\left(\pi^{0} \rightarrow \tilde{\chi}_{1}^{0} \tilde{\chi}_{1}^{0}\right)= & \left(4.64 \times 10^{-14}\right)\left(\frac{100 \mathrm{GeV}}{m_{\tilde{q}}}\right)^{4}\left(\frac{m_{\tilde{\chi}_{1}^{0}}}{1 \mathrm{MeV}}\right)^{2} \\
& \times \sqrt{1-\left(2.20 \times 10^{-4}\right)\left(\frac{m_{\tilde{\chi}_{1}^{0}}}{1 \mathrm{MeV}}\right)^{2}},(9)
\end{aligned}
$$

using $m_{\pi}=135 \mathrm{MeV}$ and $\Gamma_{\pi^{0} \text {,tot }}=7.8 \mathrm{eV}$ [9].

Since there is destructive interference between the up and down quark contributions, we consider an extreme case where the down-type squarks are so heavy that they decouple. This maximizes the branching ratio to

$$
\begin{aligned}
\operatorname{BR}\left(\pi^{0} \rightarrow \tilde{\chi}_{1}^{0} \tilde{\chi}_{1}^{0}\right)= & \left(9.30 \times 10^{-14}\right)\left(\frac{100 \mathrm{GeV}}{m_{\tilde{u}}}\right)^{4}\left(\frac{m_{\tilde{\chi}_{1}^{0}}}{1 \mathrm{MeV}}\right)^{2} \\
& \times \sqrt{1-\left(2.20 \times 10^{-4}\right)\left(\frac{m_{\tilde{\chi}_{1}^{0}}}{1 \mathrm{MeV}}\right)^{2}} .
\end{aligned}
$$

Setting the up squark masses to $100 \mathrm{GeV}$, the maximal branching ratio is $1.63 \times 10^{-10}$, when $m_{\tilde{\chi}_{1}^{0}}=55.1 \mathrm{MeV}$. The current experimental upper bound on the decay of the neutral pion to invisible particles is $2.7 \times 10^{-7}$ [55], which is at least 3 orders of magnitude greater. Hence we do not find significant constraints on supersymmetric models with extremely light binolike neutralinos from pion decays.

$$
\text { 2. } \eta \rightarrow \tilde{\chi}_{1}^{0} \tilde{\chi}_{1}^{0}, \eta^{\prime} \rightarrow \tilde{\chi}_{1}^{0} \tilde{\chi}_{1}^{0}
$$

We consider the decays of $\eta$ and $\eta^{\prime}$ mesons analogously. We take into account mixing between the $\eta^{0}$ and $\eta^{8}$ $S U$ (3)-flavor states, as described by Ref. [66], which leads 
to

$$
\begin{aligned}
\left\langle 0\left|\bar{u} \gamma^{\mu} \gamma^{5} u\right| \eta\right\rangle & =\left\langle 0\left|\bar{d} \gamma^{\mu} \gamma^{5} d\right| \eta\right\rangle \\
& =i\left(\frac{1}{\sqrt{6}} \cos \theta_{8} F_{\eta^{8}}-\frac{1}{\sqrt{3}} \sin \theta_{1} F_{\eta^{1}}\right) p_{\eta}^{\mu} \\
& \equiv i \tilde{F}_{\bar{u} u / \bar{d} d}^{\eta} p_{\eta}^{\mu}, \\
\left\langle 0\left|\bar{s} \gamma^{\mu} \gamma^{5} s\right| \eta\right\rangle & =i\left(\frac{-2}{\sqrt{6}} \cos \theta_{8} F_{\eta^{8}}-\frac{1}{\sqrt{3}} \sin \theta_{1} F_{\eta^{1}}\right) p_{\eta}^{\mu} \\
& \equiv i \tilde{F}_{\bar{s} s}^{\eta} p_{\eta}^{\mu},
\end{aligned}
$$

and

$$
\begin{aligned}
\left\langle 0\left|\bar{u} \gamma^{\mu} \gamma^{5} u\right| \eta^{\prime}\right\rangle & =\left\langle 0\left|\bar{d} \gamma^{\mu} \gamma^{5} d\right| \eta^{\prime}\right\rangle \\
& =i\left(\frac{1}{\sqrt{6}} \sin \theta_{8} F_{\eta^{8}}+\frac{1}{\sqrt{3}} \cos \theta_{1} F_{\eta^{1}}\right) p_{\eta^{\prime}}^{\mu} \\
& \equiv i \tilde{F}_{\bar{u} u / \bar{d} d}^{\eta^{\prime}} p_{\eta^{\prime}}^{\mu}, \\
\left\langle 0\left|\bar{s} \gamma^{\mu} \gamma^{5} s\right| \eta^{\prime}\right\rangle & =i\left(\frac{-2}{\sqrt{6}} \sin \theta_{8} F_{\eta^{8}}+\frac{1}{\sqrt{3}} \cos \theta_{1} F_{\eta^{1}}\right) p_{\eta^{\prime}}^{\mu} \\
& \equiv i \tilde{F}_{\bar{s} s}^{\eta^{\prime}} p_{\eta^{\prime}}^{\mu}
\end{aligned}
$$

The phenomenological values of the decay constants and mixing angles are given in Table I.

This leads to a partial decay width of

$$
\begin{aligned}
\Gamma_{\eta^{(\prime)} \rightarrow \tilde{\chi}_{1}^{0} \tilde{\chi}_{1}^{0}}= & \frac{\alpha^{2} \pi}{4} m_{\tilde{\chi}_{1}^{0}}^{2} m_{\eta^{(\prime)}} \sqrt{1-4\left(\frac{m_{\tilde{\chi}_{1}^{0}}}{m_{\eta^{(\prime)}}}\right)^{2}} \\
& \times\left[\tilde{F}_{\bar{u} u / \bar{d} d}^{\eta^{(\prime)}}\left(\frac{a_{u}^{2}}{m_{\tilde{u}_{L}}^{2}}+\frac{b_{u}^{2}}{m_{\tilde{u}_{R}}^{2}}+\frac{a_{d}^{2}}{m_{\tilde{d}_{L}}^{2}}+\frac{b_{d}^{2}}{m_{\tilde{d}_{R}}^{2}}\right)\right. \\
& \left.+\tilde{F}_{\bar{s} s}^{\eta^{(\prime)}}\left(\frac{a_{d}^{2}}{m_{\tilde{s}_{L}}^{2}}+\frac{b_{d}^{2}}{m_{\tilde{s}_{R}}^{2}}\right)\right]^{2} .
\end{aligned}
$$

If we again assume a common squark mass $m_{\tilde{q}}$, then we obtain the branching ratios:

$$
\begin{aligned}
\operatorname{BR}\left(\eta \rightarrow \tilde{\chi}_{1}^{0} \tilde{\chi}_{1}^{0}\right)= & \left(1.18 \times 10^{-15}\right)\left(\frac{100 \mathrm{GeV}}{m_{\tilde{q}}}\right)^{4}\left(\frac{m_{\tilde{\chi}_{1}^{0}}}{1 \mathrm{MeV}}\right)^{2} \\
& \times \sqrt{1-\left(1.33 \times 10^{-5}\right)\left(\frac{m_{\tilde{\chi}_{1}^{0}}}{1 \mathrm{MeV}}\right)^{2}},
\end{aligned}
$$

and

TABLE I. Input parameters for $\eta$ and $\eta^{\prime}$ decay [66].

\begin{tabular}{ll}
\hline \hline$\theta_{8}$ & $0.37 \mathrm{rad}$ \\
$\theta_{1}$ & $0.16 \mathrm{rad}$ \\
$F_{\eta^{8}} / F_{\pi}$ & 1.28 \\
$F_{\eta^{1}} / F_{\pi}$ & 1.17 \\
\hline \hline
\end{tabular}

$$
\begin{aligned}
\operatorname{BR}\left(\eta^{\prime} \rightarrow \tilde{\chi}_{1}^{0} \tilde{\chi}_{1}^{0}\right)= & \left(4.34 \times 10^{-17}\right)\left(\frac{100 \mathrm{GeV}}{m_{\tilde{q}}}\right)^{4}\left(\frac{m_{\tilde{\chi}_{1}^{0}}}{1 \mathrm{MeV}}\right)^{2} \\
& \times \sqrt{1-\left(4.36 \times 10^{-5}\right)\left(\frac{m_{\tilde{\chi}_{1}^{0}}}{1 \mathrm{MeV}}\right)^{2}} .
\end{aligned}
$$

If we decouple the strange squarks to maximize the branching ratio, we obtain for the $\eta$

$$
\begin{aligned}
\operatorname{BR}\left(\eta \rightarrow \tilde{\chi}_{1}^{0} \tilde{\chi}_{1}^{0}\right)= & \left(2.63 \times 10^{-15}\right)\left(\frac{100 \mathrm{GeV}}{m_{\tilde{q}}}\right)^{4}\left(\frac{m_{\tilde{\chi}_{1}^{0}}}{1 \mathrm{MeV}}\right)^{2} \\
& \times \sqrt{1-\left(1.33 \times 10^{-5}\right)\left(\frac{m_{\tilde{\chi}_{1}^{0}}}{1 \mathrm{MeV}}\right)^{2}} \cdot(16
\end{aligned}
$$

We use $m_{\eta}=548 \mathrm{MeV}, \quad m_{\eta^{\prime}}=958 \mathrm{MeV}, \quad \Gamma_{\eta \text {,tot }}=$ $1.30 \mathrm{keV}$, and $\Gamma_{\eta^{\prime} \text {,tot }}=205 \mathrm{keV}$, respectively [9].

Since in the case of the $\eta^{\prime}$ decay the contributions from each flavor of quark constructively interfere, we use the expression with a common squark mass, Eq. (15), to obtain a maximal branching ratio. Setting the nondecoupled squark masses to $100 \mathrm{GeV}$, the maximal branching ratios are $7.60 \times 10^{-11}$ for $\eta \rightarrow \tilde{\chi}_{1}^{0} \tilde{\chi}_{1}^{0}$ and $3.83 \times 10^{-12}$ for $\eta^{\prime} \rightarrow \tilde{\chi}_{1}^{0} \tilde{\chi}_{1}^{0}$, when $m_{\tilde{\chi}_{1}^{0}}=223 \mathrm{MeV}$ and $391 \mathrm{MeV}$, respectively. The current experimental upper bound on the decay of the $\eta$ to invisible particles is $6 \times 10^{-4}$ [9], and for the $\eta^{\prime}$ it is $1.4 \times 10^{-3}$ [9]. Thus for $\eta$ and $\eta^{\prime}$ decays we also do not find significant constraints on models with light binolike neutralinos.

\section{B. Decays of vector mesons}

We first consider the case of $\rho^{0} \rightarrow \tilde{\chi}_{1}^{0} \tilde{\chi}_{1}^{0}$, and then generalize to the other vector meson decays. Since the branching ratio depends on the ratio $m_{\tilde{\chi}_{1}^{0}} / m_{V}$ in exactly the same way for each vector meson $V$ and differs only by an overall factor, we plot the dependence once, normalized to the peak branching ratio, in Fig. 3. We note that there is no helicity suppression in this case, and so increasing the $\tilde{\chi}_{1}^{0}$

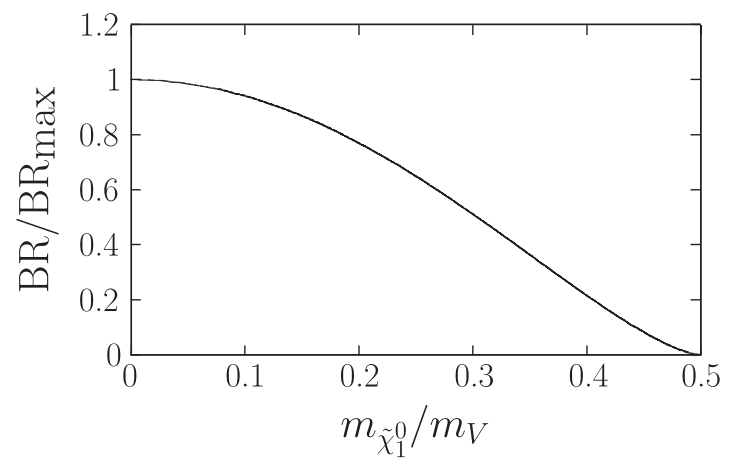

FIG. 3. The branching ratio for a vector meson decay $V \rightarrow$ $\tilde{\chi}_{1}^{0} \tilde{\chi}_{1}^{0}$ against the ratio $m_{\tilde{\chi}_{1}^{0}} / m_{V}$ normalized to the peak branching ratio. 
mass only reduces the available phase space and thus the branching ratio.

Note that ${ }^{3} S_{1}$ quarkonium states can only decay into gauginos if parity is violated. As photinos and gluinos couple to left- and right-handed squarks with equal strength, the decays ${ }^{3} S_{1}(q \bar{q}) \rightarrow \tilde{\gamma} \tilde{\gamma}$ and ${ }^{3} S_{1}(q \bar{q}) \rightarrow \tilde{g} \tilde{g}$ can only proceed if parity violation is introduced by a difference in the left- and right-handed squark masses [57]. Binos, however, couple more strongly to right-handed particles than to left-handed particles, i.e., parity is explicitly violated, and quarkonium decays into binos are possible also for degenerate left- and right-handed squark masses.

$$
\text { 1. } \rho^{0} \rightarrow \tilde{\chi}_{1}^{0} \tilde{\chi}_{1}^{0}
$$

We treat the $\rho^{0}$ as the vector equivalent of the neutral pion [i.e., the same valence quarks, $(u \bar{u}-d \bar{d}) / \sqrt{2}$ ]. The calculation of the matrix element is similar, except we use

$$
\left\langle 0\left|\bar{u} \gamma^{\mu} \gamma^{5} u\right| \rho^{0}\right\rangle=\left\langle 0\left|\bar{d} \gamma^{\mu} \gamma^{5} d\right| \rho^{0}\right\rangle=0,
$$

due to the $C P$ properties of the $\rho^{0}$ meson, and we define

$$
\left\langle 0\left|\bar{u} \gamma^{\mu} u\right| \rho^{0}\right\rangle=-\left\langle 0\left|\bar{d} \gamma^{\mu} d\right| \rho^{0}\right\rangle=\frac{i}{\sqrt{2}} m_{\rho} F_{\rho} \epsilon_{\rho}^{\mu},
$$

where $\epsilon_{\rho}^{\mu}$ is the polarization vector of the $\rho^{0}$. The factor $F_{\rho}$ we obtain by calculating the SM decay width of $\rho^{0} \rightarrow$ $e^{+} e^{-}$in this approximation to leading order in $\alpha$ :

$$
\Gamma_{\rho^{0} \rightarrow e^{+} e^{-}}=\frac{4 \pi \alpha^{2}}{3} \frac{F_{\rho}^{2}}{m_{\rho}}\left(Q_{u} H_{u}+Q_{d} H_{d}\right)^{2}=\frac{2 \pi \alpha^{2}}{3} \frac{F_{\rho}^{2}}{m_{\rho}},
$$

where $Q_{u}=2 / 3, Q_{d}=-1 / 3$ (the quark charges in units of $|e|)$ and $H_{u}=1 / \sqrt{2}, H_{d}=-1 / \sqrt{2} . H_{u, d}$ are the coefficients of the $u \bar{u}$ and $d \bar{d}$ quark field bilinears in the quark model wave function, e.g., $\rho^{0}=(u \bar{u}-d \bar{d}) / \sqrt{2} \equiv$ $\left(H_{u}^{\rho} u \bar{u}+H_{d}^{\rho} d \bar{d}\right)$; see Ref. [67] for further details. With this generic notation, Eq. (19) can easily be adjusted for other vector mesons, e.g., for $Y, H_{b}=1$, no other $H, Q_{b}=$ $-1 / 3$, so $\Gamma_{\Upsilon \rightarrow e^{+} e^{-}}=\left(4 \pi \alpha^{2} / 27\right)\left(F_{Y}^{2} / m_{\Upsilon}\right){ }^{1}$

Thus for $\rho^{0} \rightarrow \tilde{\chi}_{1}^{0} \tilde{\chi}_{1}^{0}$ we obtain for the matrix element

$$
\begin{aligned}
\mathcal{M}= & \frac{e^{2}}{8} \bar{u}_{\tilde{\chi}_{1}^{0}}\left(k_{1}\right) \gamma_{\mu} \gamma^{5} v_{\tilde{\chi}_{1}^{0}}\left(k_{2}\right)\langle 0|\left(\frac{b_{u}^{2}}{m_{\tilde{u}_{R}}^{2}}-\frac{a_{u}^{2}}{m_{\tilde{u}_{L}}^{2}}\right) \bar{u} \gamma^{\mu} u \\
& +\left(\frac{b_{d}^{2}}{m_{\tilde{d}_{R}}^{2}}-\frac{a_{d}^{2}}{m_{\tilde{d}_{L}}^{2}}\right) \bar{d} \gamma^{\mu} d\left|\rho^{0}\right\rangle-\left(k_{1} \leftrightarrow k_{2}\right) .
\end{aligned}
$$

After squaring, averaging over initial spins, and summing over final spins, we obtain

\footnotetext{
${ }^{1}$ Note that the decay of heavy-quark bound states like $J / \psi$ or $\Upsilon$ can be treated systematically in nonrelativistic QCD; see, e.g., Ref. [68].
}

$$
\begin{aligned}
\overline{|\mathcal{M}|^{2}}= & \frac{e^{4} F_{\rho}^{2}}{96}\left[\left(\frac{b_{u}^{2}}{m_{\tilde{u}_{L}}^{2}}-\frac{a_{u}^{2}}{m_{\tilde{u}_{R}}^{2}}\right)-\left(\frac{b_{d}^{2}}{m_{\tilde{d}_{L}}^{2}}-\frac{a_{d}^{2}}{m_{\tilde{d}_{R}}^{2}}\right)\right]^{2} \\
& \times\left(2 k_{1} \cdot p_{\rho} k_{2} \cdot p_{\rho}+m_{\rho}^{2} k_{1} \cdot k_{2}-3 m_{\rho}^{2} m_{\tilde{\chi}_{1}^{0}}^{2}\right),
\end{aligned}
$$

leading to the partial decay width

$$
\begin{aligned}
\Gamma_{\rho^{0} \rightarrow \tilde{\chi}_{1}^{0} \tilde{\chi}_{1}^{0}}= & \frac{\alpha^{2} \pi}{192} F_{\rho}^{2} m_{\rho}^{3}\left[1-4\left(\frac{m_{\tilde{\chi}_{1}^{0}}}{m_{\rho}}\right)^{2}\right]^{(3 / 2)} \\
& \times\left(\frac{b_{u}^{2}}{m_{\tilde{u}_{R}}^{2}}-\frac{a_{u}^{2}}{m_{\tilde{u}_{L}}^{2}}-\frac{b_{d}^{2}}{m_{\tilde{d}_{R}}^{2}}+\frac{a_{d}^{2}}{m_{\tilde{d}_{L}}^{2}}\right)^{2} \\
= & \frac{1}{32} \Gamma_{\rho^{0} \rightarrow e^{+} e^{-}} m_{\rho}^{4}\left[1-4\left(\frac{m_{\tilde{\chi}_{1}^{0}}}{m_{\rho}}\right)^{2}\right]^{(3 / 2)} \\
& \times\left(\frac{b_{u}^{2}}{m_{\tilde{u}_{R}}^{2}}-\frac{a_{u}^{2}}{m_{\tilde{u}_{L}}^{2}}-\frac{b_{d}^{2}}{m_{\tilde{d}_{R}}^{2}}+\frac{a_{d}^{2}}{m_{\tilde{d}_{L}}^{2}}\right)^{2} .
\end{aligned}
$$

If we minimize destructive interference by decoupling the left-handed up squark and the right-handed down squark, we obtain

$$
\begin{aligned}
\operatorname{BR}\left(\rho^{0} \rightarrow \tilde{\chi}_{1}^{0} \tilde{\chi}_{1}^{0}\right)= & \left(8.01 \times 10^{-15}\right)\left(\frac{100 \mathrm{GeV}}{m_{\tilde{q}}}\right)^{4} \\
& \times\left[1-\left(6.65 \times 10^{-6}\right)\left(\frac{m_{\tilde{\chi}_{1}^{0}}}{1 \mathrm{MeV}}\right)^{2}\right]^{(3 / 2)},
\end{aligned}
$$

using $m_{\rho}=775 \mathrm{MeV}, \quad \Gamma_{\rho^{0} \rightarrow e^{+} e^{-}} / \Gamma_{\rho^{0}, \text { tot }}=4.71 \times 10^{-5}$ [9]. The branching ratio is maximized for $m_{\tilde{\chi}_{1}^{0}}=0$. Setting the nondecoupled squark masses to $100 \mathrm{GeV}$, we find a branching ratio of $8.01 \times 10^{-15}$. We can find no experimental bound at this time for the branching ratio of $\rho^{0} \rightarrow$ invisible, but note that a branching ratio of $10^{-14}$ is far below the bounds on other branching ratios measured for the $\rho^{0}$ meson [9].

$$
\text { 2. } \omega \rightarrow \tilde{\chi}_{1}^{0} \tilde{\chi}_{1}^{0}, \phi \rightarrow \tilde{\chi}_{1}^{0} \tilde{\chi}_{1}^{0}
$$

The mixing between the $S U(3)$-flavor states among the vector mesons is very different from the pseudoscalars. Conveniently, it is such that the $\phi$ meson is nearly pure $s \bar{s}$ and the $\omega$ is $(u \bar{u}+d \bar{d}) / \sqrt{2}$, and we treat them as these pure states.

This means that the decay $\omega \rightarrow \tilde{\chi}_{1}^{0} \tilde{\chi}_{1}^{0}$ is analogous to $\rho \rightarrow \tilde{\chi}_{1}^{0} \tilde{\chi}_{1}^{0}$, taking into account the constructive rather than destructive interference between the up and down quarks:

$$
\begin{aligned}
\Gamma_{\omega \rightarrow \tilde{\chi}_{1}^{0} \tilde{\chi}_{1}^{0}}= & \frac{9}{32} \Gamma_{\omega \rightarrow e^{+} e^{-}} m_{\omega}^{4}\left[1-4\left(\frac{m_{\tilde{\chi}_{1}^{0}}}{m_{\omega}}\right)^{2}\right]^{(3 / 2)} \\
& \times\left(\frac{b_{u}^{2}}{m_{\tilde{u}_{R}}^{2}}-\frac{a_{u}^{2}}{m_{\tilde{u}_{L}}^{2}}+\frac{b_{d}^{2}}{m_{\tilde{d}_{R}}^{2}}-\frac{a_{d}^{2}}{m_{\tilde{d}_{L}}^{2}}\right)^{2}
\end{aligned}
$$

If we take a conservative estimate by decoupling the left-handed up and down squark masses, we obtain 


$$
\begin{aligned}
\operatorname{BR}\left(\omega \rightarrow \tilde{\chi}_{1}^{0} \tilde{\chi}_{1}^{0}\right)= & \left(1.57 \times 10^{-13}\right)\left(\frac{100 \mathrm{GeV}}{m_{\tilde{q}_{R}}}\right)^{4} \\
& \times\left[1-\left(6.53 \times 10^{-6}\right)\left(\frac{m_{\tilde{\chi}_{1}^{0}}}{1 \mathrm{MeV}}\right)^{2}\right]^{(3 / 2)},
\end{aligned}
$$

with $m_{\omega}=783 \mathrm{MeV}$, and $\Gamma_{\omega \rightarrow e^{+} e^{-}} / \Gamma_{\omega, \text { tot }}=7.16 \times 10^{-5}$ [9]. This branching ratio is maximized for $m_{\tilde{\chi}_{1}^{0}}=0$. Setting the nondecoupled squark masses to $100 \mathrm{GeV}$, we find $\operatorname{BR}\left(\omega \rightarrow \tilde{\chi}_{1}^{0} \tilde{\chi}_{1}^{0}\right)=1.57 \times 10^{-13}$.

The $\phi$ case is particularly simple. The partial width is given by

$$
\begin{aligned}
\Gamma_{\phi \rightarrow \tilde{\chi}_{1}^{0} \tilde{\chi}_{1}^{0}}= & \frac{9}{32} \Gamma_{\phi \rightarrow e^{+} e^{-}} m_{\phi}^{4}\left[1-4\left(\frac{m_{\tilde{\chi}_{1}^{0}}}{m_{\phi}}\right)^{2}\right]^{(3 / 2)} \\
& \times\left(\frac{b_{d}^{2}}{m_{\tilde{S}_{R}}^{2}}-\frac{a_{d}^{2}}{m_{\tilde{s}_{L}}^{2}}\right)^{2} .
\end{aligned}
$$

If we minimize destructive interference by decoupling the left-handed strange squark mass, we obtain

$$
\begin{aligned}
\operatorname{BR}\left(\phi \rightarrow \tilde{\chi}_{1}^{0} \tilde{\chi}_{1}^{0}\right)= & \left(7.51 \times 10^{-14}\right)\left(\frac{100 \mathrm{GeV}}{m_{\tilde{s}_{R}}}\right)^{4} \\
& \times\left[1-\left(3.85 \times 10^{-6}\right)\left(\frac{m_{\tilde{\chi}_{1}^{0}}}{1 \mathrm{MeV}}\right)^{2}\right]^{(3 / 2)},
\end{aligned}
$$

which is maximized for $m_{\tilde{\chi}_{1}^{0}}=0$. Here $m_{\phi}=1.02 \mathrm{GeV}$, and $\Gamma_{\phi \rightarrow e^{+} e^{-}} / \Gamma_{\phi, \text { tot }}=2.97 \times 10^{-4}$ [9]. Setting the righthanded strange squark mass to $100 \mathrm{GeV}$, we find a branching ratio of $7.51 \times 10^{-14}$. Again, we can find no experimental bound at this time for the branching ratios of $\phi$ or $\omega \rightarrow$ invisible, but note that branching ratios of $10^{-13}$ and $10^{-14}$ are far below the bounds on other branching ratios measured for these mesons [9].

$$
\text { 3. } J / \psi \rightarrow \tilde{\chi}_{1}^{0} \tilde{\chi}_{1}^{0} \text {, } \rightarrow \tilde{\chi}_{1}^{0} \tilde{\chi}_{1}^{0}
$$

The squarks associated with the heavier flavors of quarks (charm, bottom, and top) are expected to mix their left-handed and right-handed components much more than the other flavors $[2,3]$. We take this into account for the $J / \psi$ and $Y$ decays. Writing the mass eigenstates of the charm squarks $\tilde{c}_{1,2}$ as

$$
\left(\begin{array}{c}
\tilde{c}_{1} \\
\tilde{c}_{2}
\end{array}\right)=\left(\begin{array}{cc}
\cos \theta_{\tilde{c}} & -\sin \theta_{\tilde{c}} \\
\sin \theta_{\tilde{c}} & \cos \theta_{\tilde{c}}
\end{array}\right)\left(\begin{array}{c}
\tilde{c}_{L} \\
\tilde{c}_{R}
\end{array}\right),
$$

we obtain

$$
\begin{aligned}
\mathcal{M}= & \frac{1}{i}\left\langle\tilde{\chi}_{1}^{0} \tilde{\chi}_{1}^{0}\right|\left\{\tilde{\tilde{\chi}}_{1}^{0}\left[i|e| a_{u}\left(\cos \theta_{\tilde{c}} \tilde{c}_{1}^{*}+\sin \theta_{\tilde{c}} \tilde{c}_{2}^{*}\right) P_{L}+i|e| b_{u}\left(\cos \theta_{\tilde{c}} \tilde{c}_{2}^{*}-\sin \theta_{\tilde{c}} \tilde{c}_{1}^{*}\right) P_{R}\right] c\right\}\left\{\overline { c } \left[-i|e| a_{u}\left(\cos \theta_{\tilde{c}} \tilde{c}_{1}+\sin \theta_{\tilde{c}} \tilde{c}_{2}\right) P_{R}\right.\right. \\
& \left.\left.-i|e| b_{u}\left(\cos \theta_{\tilde{c}} \tilde{c}_{2}-\sin \theta_{\tilde{c}} \tilde{c}_{1}\right) P_{L}\right] \tilde{\chi}_{1}^{0}\right\}|J / \psi\rangle \\
= & \frac{e^{2} F_{J / \psi} m_{J / \psi}}{8}\left[\bar{u}_{\tilde{\chi}_{1}^{0}}\left(k_{1}\right) \xi_{J / \psi} \gamma^{5} v_{\tilde{\chi}_{1}^{0}}\left(k_{2}\right)-\bar{u}_{\tilde{\chi}_{1}^{0}}\left(k_{2}\right) \xi_{J / \psi} \gamma^{5} v_{\tilde{\chi}_{1}^{0}}\left(k_{1}\right)\right] \\
& \times\left(\frac{\left[a_{u} \cos \theta_{\tilde{c}}\right]^{2}-\left[b_{u} \sin \theta_{\tilde{c}}\right]^{2}}{m_{\tilde{c}_{1}}^{2}}+\frac{\left[a_{u} \sin \theta_{\tilde{c}}\right]^{2}-\left[b_{u} \cos \theta_{\tilde{c}}\right]^{2}}{m_{\tilde{c}_{2}}^{2}}\right)
\end{aligned}
$$

since $\left\langle 0\left|\bar{c} P_{L / R} c\right| J / \psi\right\rangle=0$. Hence

$$
\begin{aligned}
\overline{|\mathcal{M}|^{2}}= & \frac{e^{4} F_{J / \psi}^{2}}{48}\left(\frac{\left(a_{u} \cos \theta_{\tilde{c}}\right)^{2}-\left(b_{u} \sin \theta_{\tilde{c}}\right)^{2}}{m_{\tilde{c}_{1}}^{2}}+\frac{\left(a_{u} \sin \theta_{\tilde{c}}\right)^{2}-\left(b_{u} \cos \theta_{\tilde{c}}\right)^{2}}{m_{\tilde{c}_{2}}^{2}}\right)^{2} \\
& \times\left(2 k_{1} \cdot p_{J / \psi} k_{2} \cdot p_{J / \psi}+m_{J / \psi}^{2} k_{1} \cdot k_{2}-3 m_{J / \psi}^{2} m_{\tilde{\chi}_{1}^{0}}^{2}\right),
\end{aligned}
$$

and so we obtain

$$
\Gamma_{J / \psi \rightarrow \tilde{\chi}_{1}^{0} \tilde{\chi}_{1}^{0}}=\frac{9}{128} \Gamma_{J / \psi \rightarrow e^{+} e^{-}} m_{J / \psi}^{4}\left[1-4\left(\frac{m_{\tilde{\chi}_{1}^{0}}}{m_{J / \psi}}\right)^{2}\right]^{(3 / 2)}\left(\frac{\left(a_{u} \cos \theta_{\tilde{c}}\right)^{2}-\left(b_{u} \sin \theta_{\tilde{c}}\right)^{2}}{m_{\tilde{c}_{1}}^{2}}+\frac{\left(a_{u} \sin \theta_{\tilde{c}}\right)^{2}-\left(b_{u} \cos \theta_{\tilde{c}}\right)^{2}}{m_{\tilde{c}_{2}}^{2}}\right)^{2} .
$$

Our results agree with the decay width for orthotoponium decay to a photino pair in Ref. [57], with the replacements $a_{u / d}, b_{u / d} \rightarrow e_{u}=2 / 3, \theta_{\tilde{c} / \tilde{b}} \rightarrow \tilde{\theta}_{t}$, and $m_{\tilde{c} / \tilde{b}} \rightarrow m_{\tilde{t}}$ for the case $m_{\tilde{\gamma}} \rightarrow 0$, and assuming $m_{\theta}=2 m_{t}$.

The greatest branching ratio occurs when $\theta_{\tilde{c}}=\pi / 2$ and the heavier charm squark is decoupled. In this limit, and using $m_{J / \psi}=3.10 \mathrm{GeV}, \Gamma_{J / \psi \rightarrow e^{+} e^{-}} / \Gamma_{J / \psi \text {,tot }}=5.94 \times 10^{-2}$ [9], the branching ratio for $J / \psi \rightarrow \tilde{\chi}_{1}^{0} \tilde{\chi}_{1}^{0}$ is 


$$
\mathrm{BR}\left(J / \psi \rightarrow \tilde{\chi}_{1}^{0} \tilde{\chi}_{1}^{0}\right)=\left(5.12 \times 10^{-9}\right)\left(\frac{100 \mathrm{GeV}}{m_{\tilde{c}_{1}}}\right)^{4}\left[1-\left(4.17 \times 10^{-7}\right)\left(\frac{m_{\tilde{\chi}_{1}^{0}}}{1 \mathrm{MeV}}\right)^{2}\right]^{(3 / 2)},
$$

which is maximized for $m_{\tilde{\chi}_{1}^{0}}=0$. Setting $m_{\tilde{c}_{1}}$ to $100 \mathrm{GeV}$, we find a branching ratio of $5.12 \times 10^{-9}$. This is well below the experimental upper bound of $5.9 \times 10^{-4}$ [69], but not very much below the branching ratio for the SM process $J / \psi \rightarrow \nu \bar{\nu}$ of $2.70 \times 10^{-8}[61]$.

Analogously,

$$
\Gamma_{\Upsilon \rightarrow \tilde{\chi}_{1}^{0} \tilde{\chi}_{1}^{0}}=\frac{9}{32} \Gamma_{\Upsilon \rightarrow e^{+} e^{-}} m_{\Upsilon}^{4}\left[1-4\left(\frac{m_{\tilde{\chi}_{1}^{0}}}{m_{\Upsilon}}\right)^{2}\right]^{(3 / 2)}\left(\frac{\left(a_{d} \cos \theta_{\tilde{b}}\right)^{2}-\left(b_{d} \sin \theta_{\tilde{b}}\right)^{2}}{m_{\tilde{b}_{1}}^{2}}+\frac{\left(a_{d} \sin \theta_{\tilde{b}}\right)^{2}-\left(b_{d} \cos \theta_{\tilde{b}}\right)^{2}}{m_{\tilde{b}_{2}}^{2}}\right)^{2} .
$$

Here $\theta_{\tilde{b}}$ is the mixing angle in the bottom squark sector. The greatest branching ratio occurs when $\theta_{\tilde{b}}=\pi / 2$ and the heavier bottom squark is infinitely heavy. In this limit, the branching ratio for $Y \rightarrow \tilde{\chi}_{1}^{0} \tilde{\chi}_{1}^{0}$ is

$$
\mathrm{BR}\left(\mathrm{Y} \rightarrow \tilde{\chi}_{1}^{0} \tilde{\chi}_{1}^{0}\right)=\left(4.47 \times 10^{-8}\right)\left(\frac{100 \mathrm{GeV}}{m_{\tilde{b}_{1}}}\right)^{4}\left[1-\left(4.69 \times 10^{-8}\right)\left(\frac{m_{\tilde{\chi}_{1}^{0}}}{1 \mathrm{MeV}}\right)^{2}\right]^{(3 / 2)},
$$

which is maximized for $m_{\tilde{\chi}_{1}^{0}}=0$. Here we have used $m_{Y}=9.46 \mathrm{GeV}$, and $\Gamma_{Y \rightarrow e^{+} e^{-}} / \Gamma_{Y, \text { tot }}=2.38 \times 10^{-2}[9]$. Setting the lighter bottom squark mass $m_{\tilde{b}_{1}}$ to $100 \mathrm{GeV}$, we find a branching ratio of $4.47 \times 10^{-8}$, which is well below the experimental upper bound of $2.5 \times 10^{-3}$ [70], and also well below the branching ratio for the SM process $Y \rightarrow \nu \bar{\nu}$ of $1.05 \times 10^{-5}$ [61].

We note that $Y \rightarrow$ invisible has been used to probe light dark matter through inversion of the annihilation cross section [47].

\section{III. $K^{-} \rightarrow \pi^{-} \tilde{\chi}_{1}^{0} \tilde{\chi}_{1}^{0}$ AND $B^{-} \rightarrow K^{-} \tilde{\chi}_{1}^{0} \tilde{\chi}_{1}^{0}$ WITHIN THE MINIMAL FLAVOR-VIOLATING MSSM}

As mentioned in Sec. II A, the two-body decays of pseudoscalar mesons all have widths proportional to the square of the mass of the $\tilde{\chi}_{1}^{0}$, and so there are no bounds for the case of massless neutralinos. This is not the case for three-body final states, and so we examine whether there are significant bounds from the decays $K^{-} \rightarrow \pi^{-} \tilde{\chi}_{1}^{0} \tilde{\chi}_{1}^{0}$ and $B^{-} \rightarrow K^{-} \tilde{\chi}_{1}^{0} \tilde{\chi}_{1}^{0}$, where the SM analogs, $K^{-} \rightarrow \pi^{-} \nu \bar{\nu}$ and $B^{-} \rightarrow K^{-} \nu \bar{\nu}$, have very low branching ratios [71-74].

We present the results for the case of massless neutralinos. The phase space is smaller as the $\tilde{\chi}_{1}^{0}$ mass increases, and any terms in the matrix-element-squared proportional to the $\tilde{\chi}_{1}^{0}$ mass are subdominant.

\section{A. General analysis}

For this section, we impose MFV [39] at the low-energy scale at which we are working (i.e., at the mass of the decaying meson). MFV models are defined by the requirement that all flavor-violating and $C P$-violating transitions are described by the Cabibbo-Kobayashi-Maskawa (CKM) matrix of the SM; see e.g., Refs. [75,76] and references therein. Thus the decays $K^{-} \rightarrow \pi^{-} \tilde{\chi}_{1}^{0} \tilde{\chi}_{1}^{0}$ and $B^{-} \rightarrow$ $K^{-} \tilde{\chi}_{1}^{0} \tilde{\chi}_{1}^{0}$ only have leading contributions from one-loop diagrams. Those for $K^{-} \rightarrow \pi^{-} \tilde{\chi}_{1}^{0} \tilde{\chi}_{1}^{0}$ are shown in Figs. 47 , without the diagrams with the neutralinos crossed. Through the loops, the partial width depends on many parameters, and not in a simple way. Therefore we have opted to use a set of supersymmetric benchmark scenarios, thereby fixing the masses and couplings. We use the masses and mixings of the Snowmass points SPS1a, SPS2, SPS3, SPS4, and SPS5 [77], calculated using SOFTSUSY [78]. We have modified the neutralino sector of these benchmark points by choosing $M_{1}$ according to Eq. (3) such that the lightest neutralino is binolike and massless. The resulting supersymmetric models we denote as "pseudo-SPS" points. The imposition of MFV and the degeneracy of the up- and charm-squark masses leads to a super-Glashow-Iliopoulos-Maiani [79] suppression in the $K^{-} \rightarrow \pi^{-} \tilde{\chi}_{1}^{0} \tilde{\chi}_{1}^{0}$ decay and to a lesser extent in the $B^{-} \rightarrow$ $K^{-} \tilde{\chi}_{1}^{0} \tilde{\chi}_{1}^{0}$ decay.

Since the scale of the sparticle masses and the $W$ boson mass are so much greater than the masses of the kaon or $B$ meson, we integrate out the heavy degrees of freedom to obtain effective four-fermion vertices [80]. We then write the amplitude for $K^{-} \rightarrow \pi^{-} \tilde{\chi}_{1}^{0} \tilde{\chi}_{1}^{0}$ as

$$
\begin{aligned}
\mathcal{M}_{K^{-} \rightarrow \pi^{-} \tilde{\chi}_{1}^{0} \tilde{\chi}_{1}^{0}}^{\mathrm{MFV}}= & \frac{G_{F}}{2}\left(C_{\not b^{+}} \bar{u}\left(k_{1}\right) \gamma_{\mu} \gamma^{5} v\left(k_{2}\right)\left\langle\pi^{-}\left|\bar{d} \gamma^{\mu} s\right| K^{-}\right\rangle+\frac{C_{\not p}}{m_{K}} \bar{u}\left(k_{1}\right) \not p_{s} v\left(k_{2}\right)\left\langle\pi^{-}|\bar{d} s| K^{-}\right\rangle\right. \\
& \left.+C_{L} \bar{u}\left(k_{1}\right) P_{L} v\left(k_{2}\right)\left\langle\pi^{-}|\bar{d} s| K^{-}\right\rangle+C_{R} \bar{u}\left(k_{1}\right) P_{R} v\left(k_{2}\right)\left\langle\pi^{-}|\bar{d} s| K^{-}\right\rangle\right)-\left(k_{1} \leftrightarrow k_{2}\right),
\end{aligned}
$$




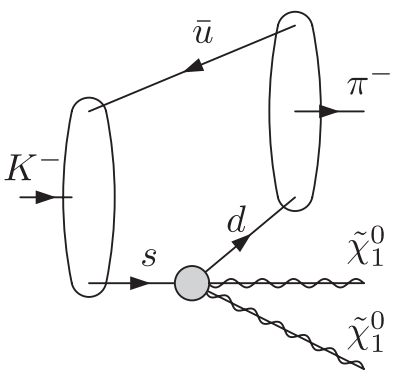

FIG. 4. Generic Feynman diagram of the decay $K^{-} \rightarrow$ $\pi^{-} \tilde{\chi}_{1}^{0} \tilde{\chi}_{1}^{0}$.

recalling that $k_{1}$ and $k_{2}$ are the momenta of the (indistinguishable) final-state neutralinos. The $C_{i}$ coefficients, $i=$ $\not F, \not p, L$, and $R$, are dimensionless. These are the only terms possible, since $\overline{\tilde{\chi}}_{1}^{0} \gamma^{\mu} \tilde{\chi}_{1}^{0}$ and $\overline{\tilde{\chi}}_{1}^{0}\left[\gamma^{\mu}, \gamma^{\nu}\right] \tilde{\chi}_{1}^{0}$ are identically zero for Majorana fermions. Conservation of momentum and the Dirac equation for the spinors can be used to reduce any other terms to the set above [applying the identities $\bar{u}\left(k_{2}\right) \gamma^{\mu} \gamma^{5} v\left(k_{1}\right)=v^{T}\left(k_{2}\right) \gamma^{5} \gamma^{T \mu} \bar{u}^{T}\left(k_{2}\right)=$ $-\bar{u}\left(k_{1}\right) \gamma^{\mu} \gamma^{5} v\left(k_{2}\right) \quad$ and $\quad \bar{u}\left(k_{2}\right) P_{L / R} v\left(k_{1}\right)=$ $\left.v^{T}\left(k_{2}\right) P_{L / R} \bar{u}^{T}\left(k_{2}\right)=-\bar{u}\left(k_{1}\right) P_{L / R} v\left(k_{2}\right)\right]$.

The coefficients $C_{i}$ were calculated with the aid of FORMCALC [81], using techniques detailed in Ref. [82], with the approximation of neglecting all external momenta compared to the sparticle and $W$ boson masses. The loop integrals in this approximation are straightforward and can be written as powers and logarithms of masses, so numerical instabilities in the reduction of tensor integrals with extreme scale differences are avoided. Variations of these
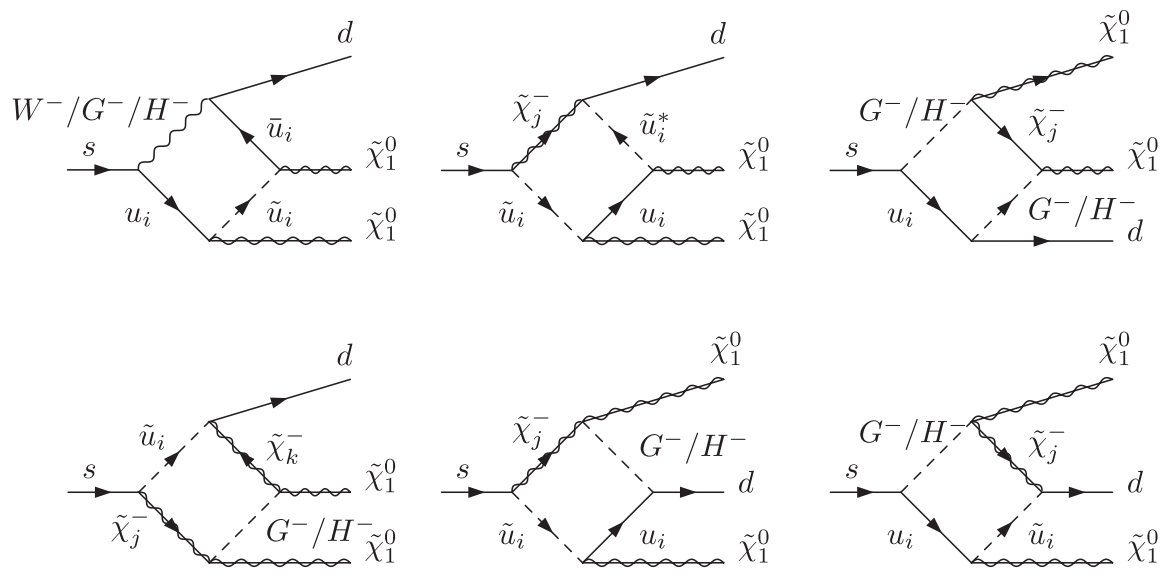

FIG. 5. Box Feynman diagrams for the decay $s \rightarrow d \tilde{\chi}_{1}^{0} \tilde{\chi}_{1}^{0}$.
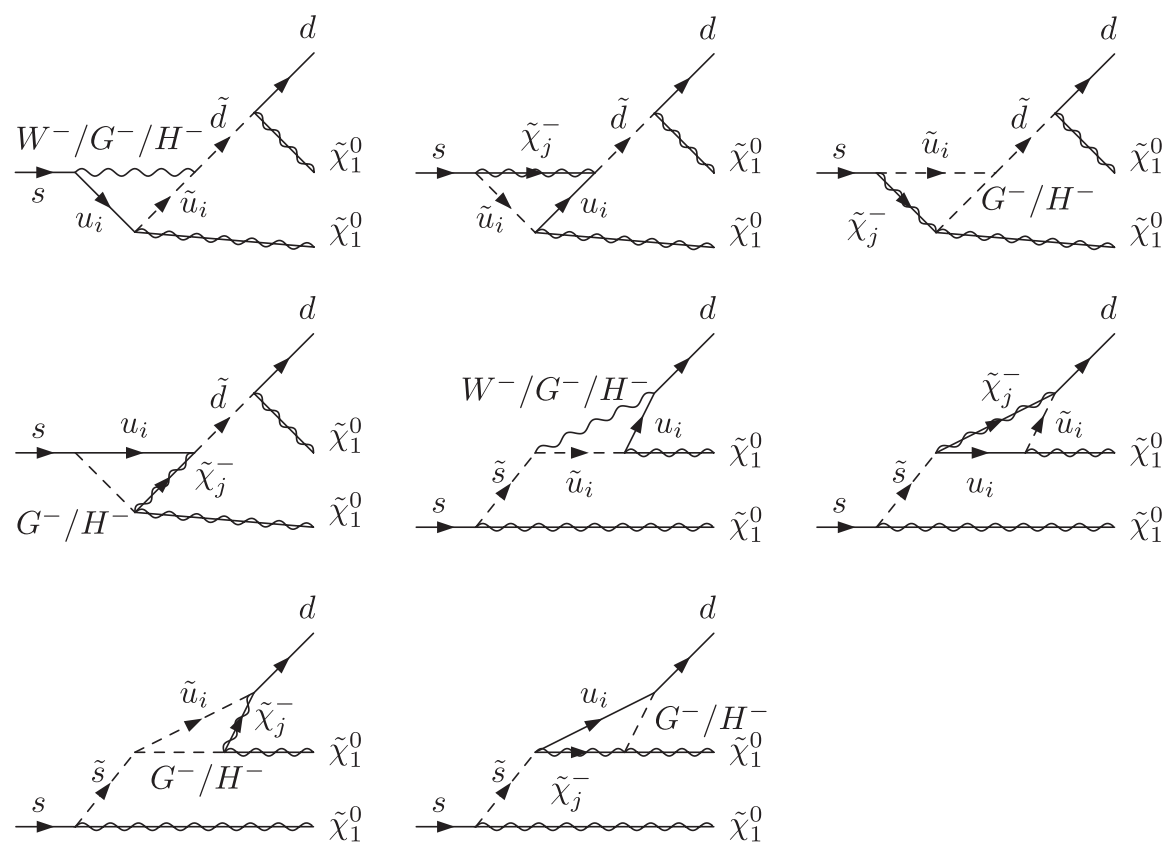

FIG. 6. Triangle Feynman diagrams for the decay $s \rightarrow d \tilde{\chi}_{1}^{0} \tilde{\chi}_{1}^{0}$. 

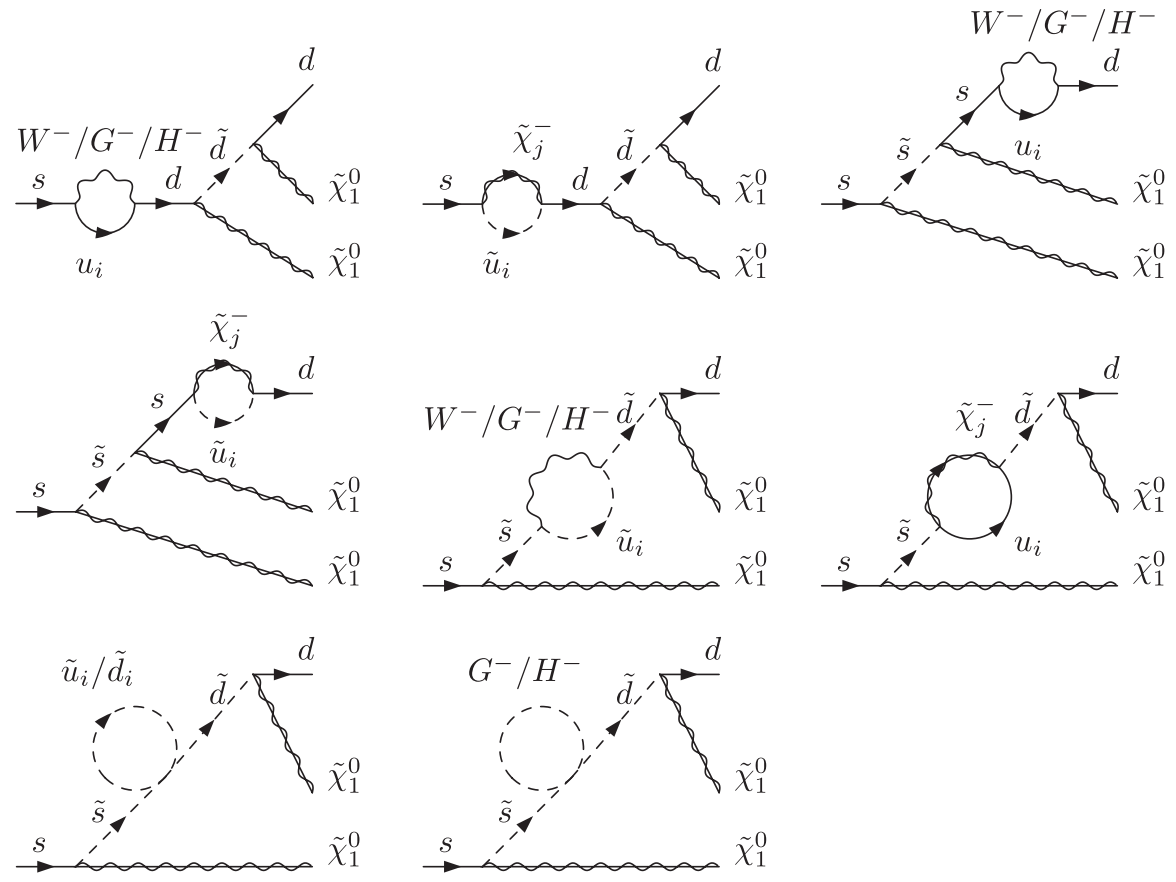

FIG. 7. Circle Feynman diagrams for the decay $s \rightarrow d \tilde{\chi}_{1}^{0} \tilde{\chi}_{1}^{0}$.

coefficients over the phase space of the decay are of order $m_{K}^{2} / m_{W}^{2}$ and therefore we ignore them. This is analogous to the approach taken in Ref. [83] in calculating the leading order value for the standard model decay $K^{+} \rightarrow \pi^{0} e^{+} \nu$.

Our calculation takes into account all diagrams at the one-loop level and is not restricted by requiring the squark masses to be small compared to the mass of the $W$ boson, thus improving the original calculations for photinolike $\tilde{\chi}_{1}^{0}$ of Refs. [34,35].

The relevant terms for $B^{-} \rightarrow K^{-} \tilde{\chi}_{1}^{0} \tilde{\chi}_{1}^{0}$ can be obtained from the above by replacing the mesons in the bra and ket appropriately and the $s$ quark with a $b$ quark, and the $\bar{d}$ with a $\bar{s}$.

We use the mesonic form factors as given in Ref. [84] for $K^{-} \rightarrow \pi^{-} \tilde{\chi}_{1}^{0} \tilde{\chi}_{1}^{0}$ and Refs. [85,86] for $B^{-} \rightarrow K^{-} \tilde{\chi}_{1}^{0} \tilde{\chi}_{1}^{0}$ (reproduced for convenience in the Appendix), but for the moment, we shall use the shorthand for the mesonic current $\left\langle\pi^{-}\left|\bar{d} \gamma^{\mu} s\right| K^{-}\right\rangle=F^{\mu}$. Taking the dot product of both sides with the difference of the momenta of the kaon and the pion, which to a good approximation is equal to the difference in momenta of the strange quark and the down quark, and using the Dirac equation for the quarks allows us to write

$$
\left\langle\pi^{-}|\bar{d} s| K^{-}\right\rangle=\frac{F \cdot\left(p_{K}-p_{\pi}\right)}{\left(m_{s}+m_{d}\right)} .
$$

In this notation

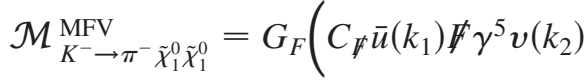

$$
\begin{aligned}
& +C_{\not p} \bar{u}\left(k_{1}\right) \not p_{s} \gamma^{5} v\left(k_{2}\right) \frac{F \cdot\left(p_{K}-p_{\pi}\right)}{m_{K}\left(m_{s}+m_{d}\right)} \\
& +C_{L} \bar{u}\left(k_{1}\right) P_{L} v\left(k_{2}\right) \frac{F \cdot\left(p_{K}-p_{\pi}\right)}{\left(m_{s}+m_{d}\right)} \\
& \left.+C_{R} \bar{u}\left(k_{1}\right) P_{R} v\left(k_{2}\right) \frac{F \cdot\left(p_{K}-p_{\pi}\right)}{\left(m_{s}+m_{d}\right)}\right) .
\end{aligned}
$$

As can be seen in Sec. III B, the coefficient $C_{\not \mid t}$ is much larger than the other coefficients for the benchmark points we use for the decay $K^{-} \rightarrow \pi^{-} \tilde{\chi}_{1}^{0} \tilde{\chi}_{1}^{0}$. If we neglect all the $C$ coefficients except $C_{\not \mathscr{H}}$, we find that the spin-averaged matrix element squared for $K^{-} \rightarrow \pi^{-} \tilde{\chi}_{1}^{0} \tilde{\chi}_{1}^{0}$ is

$$
\begin{aligned}
\overline{\mid \mathcal{M}_{K^{-} \rightarrow \pi^{-} \tilde{\chi}_{1}^{0} \tilde{\chi}_{1}^{2}}^{\mathrm{MFV}}=} & 4 G_{F}^{2}\left|C_{\not \mid l}\right|^{2}\left(F^{*} \cdot k_{1} F \cdot k_{2}+F^{*} \cdot k_{2} F \cdot k_{1}\right. \\
& \left.-F^{*} \cdot F k_{1} \cdot k_{2}\right),
\end{aligned}
$$

which differs from that for $K^{-} \rightarrow \pi^{0} e \bar{\nu}$ (which has a branching ratio of $5.08 \times 10^{-2}$ [9]) only by an overall factor [assuming a massless electron and isospin symmetry for the up and down valence quarks; the factor of $(1 / \sqrt{2})^{2}$ from the coefficient of $u \bar{u}$ in $\pi^{0}$ balances the factor of $1 / 2$ from identical final-state particles in the $K^{-} \rightarrow \pi^{-} \tilde{\chi}_{1}^{0} \tilde{\chi}_{1}^{0}$ case]. In this case the behavior over the phase space is identical and the ratio of the decay widths is equal to the ratio of $\left|C_{\not \mid l}\right|^{2}$ to $\left|V_{u s}\right|^{2}$. For the case of $B^{-} \rightarrow K^{-} \tilde{\chi}_{1}^{0} \tilde{\chi}_{1}^{0}$, we 


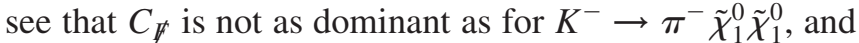
we present the results from directly evaluating the decay width using the expressions for $F^{\mu}$ given in Refs. [85,86] (though with diminished accuracy from using mesonic form factors). Direct evaluation was found to agree at the percent level for $K^{-} \rightarrow \pi^{-} \tilde{\chi}_{1}^{0} \tilde{\chi}_{1}^{0}$. For $B^{-} \rightarrow K^{-} \tilde{\chi}_{1}^{0} \tilde{\chi}_{1}^{0}, C_{R}$ is comparable to $C_{\not H^{*}}$ and the effect of neglecting it decreases the branching ratio from by roughly $0.3 \%$ for pseudo-SPS5 up to by $66 \%$ for pseudo-SPS4.

\section{B. Numerical results}

We present the numerical values of the $C$ coefficients in Tables II and III. In the final column of each table, headed $\mathrm{BR} / \exp$, we present the ratio of either the computed $K^{-} \rightarrow \pi^{-} \tilde{\chi}_{1}^{0} \tilde{\chi}_{1}^{0}$ branching ratio to the experimental value of the branching ratio for $K^{-} \rightarrow \pi^{-} \nu \bar{\nu}$ (which is $1.73 \times$ $10^{-10}$ [87]), or the computed branching ratio of $B^{-} \rightarrow$ $K^{-} \tilde{\chi}_{1}^{0} \tilde{\chi}_{1}^{0}$ to the current experimental upper bound on the branching ratio for $B^{-} \rightarrow K^{-} \nu \bar{\nu}$ (which is $1.4 \times 10^{-5}$ [74]).

We see that the branching ratios for the decays $K^{-} \rightarrow$ $\pi^{-} \tilde{\chi}_{1}^{0} \tilde{\chi}_{1}^{0}$ and $B^{-} \rightarrow K^{-} \tilde{\chi}_{1}^{0} \tilde{\chi}_{1}^{0}$ in the MSSM with MFV are several orders of magnitude smaller than the respective standard model processes, with no chance to observe them in the experiments of today and the foreseeable future.

The strong relative suppression of the kaon and $B$ meson decays to neutralinos compared to their respective SM decays to neutrinos can be explained as a combination of various factors. First, the sparticle masses of the SPS benchmark scenarios are considerably larger than the $W$ mass; in particular, the SPS2 benchmark point with squark masses of $\mathcal{O}(1.5) \mathrm{TeV}$ is most strongly suppressed. Moreover, the SM process has four $S U(2)$ vertices while the MSSM process has two $S U(2)$ vertices and two $U(1)$ vertices, resulting in a further suppression of the MSSM decay matrix element proportional to $\tan ^{2} \theta_{W}$. Since the flavor-changing aspect is carried by the $W$ boson or the charged Higgs bosons, the quarks/squarks are mainly left handed, and their couplings to the binolike neutralinos are suppressed by their small hypercharge. Finally, the spin structure of the MSSM decay amplitude provides another order-of-magnitude suppression with respect to the SM decay. Considering all these factors together leads to a suppression of several orders of magnitude.

\section{IV. $K^{-} \rightarrow \pi^{-} \tilde{\chi}_{1}^{0} \tilde{\chi}_{1}^{0}$ AND $B^{-} \rightarrow K^{-} / \pi^{-} \tilde{\chi}_{1}^{0} \tilde{\chi}_{1}^{0}$ WITHIN THE NONMINIMAL FLAVOR-VIOLATING MSSM}

Going beyond the previous section, we here consider nonminimal flavor violation in the MSSM, for which there are many potential sources. For example, the squark mass matrix in the soft-breaking part of the Lagrangian in general couples squarks of different flavor to each other, resulting in flavor-changing neutral currents (FCNCs). Thus from the theoretical point of view there is a priori no reason to assume MFV within the MSSM.

To deal with the complicated flavor structure of the MSSM, we employ the mass insertion approximation [76,88], which is defined in the super-CKM basis $[76,88,89]$. In this basis, the Lagrangian is written in the quark mass eigenstate basis. The squarks are rotated with the same rotation matrices as the quarks. This leads to a

TABLE II. Numerical values for the coefficients $C_{i}$ defined in Eq. (35) for $K^{-} \rightarrow \pi^{-} \tilde{\chi}_{1}^{0} \tilde{\chi}_{1}^{0}$ at the various pseudo-SPS points described in the text. The final column shows the ratio of the branching ratio for $K^{-} \rightarrow \pi^{-} \tilde{\chi}_{1}^{0} \tilde{\chi}_{1}^{0}$ to the experimental value of the branching ratio for $K^{-} \rightarrow \pi^{-} \nu \bar{\nu}\left(1.73 \times 10^{-10}\right)$.

\begin{tabular}{|c|c|c|c|c|c|c|}
\hline Pseudo- SPS & $\left|C_{\not h}\right|$ & $\left|C_{p}\right|$ & $\left|C_{L}\right|$ & $\left|C_{R}\right|$ & BR & $\mathrm{BR} / \exp$ \\
\hline 1a & $5.70 \times 10^{-8}$ & $7.45 \times 10^{-14}$ & $4.93 \times 10^{-12}$ & $2.60 \times 10^{-10}$ & $3.28 \times 10^{-16}$ & $1.90 \times 10^{-6}$ \\
\hline 2 & $3.81 \times 10^{-9}$ & $7.77 \times 10^{-14}$ & $9.55 \times 10^{-12}$ & $4.10 \times 10^{-11}$ & $1.47 \times 10^{-18}$ & $8.49 \times 10^{-9}$ \\
\hline 3 & $2.63 \times 10^{-8}$ & $3.74 \times 10^{-14}$ & $5.13 \times 10^{-12}$ & $1.25 \times 10^{-10}$ & $6.99 \times 10^{-17}$ & $4.04 \times 10^{-7}$ \\
\hline 4 & $2.95 \times 10^{-8}$ & $6.41 \times 10^{-14}$ & $1.34 \times 10^{-12}$ & $1.42 \times 10^{-9}$ & $8.76 \times 10^{-17}$ & $5.06 \times 10^{-7}$ \\
\hline 5 & $7.12 \times 10^{-8}$ & $2.81 \times 10^{-14}$ & $5.61 \times 10^{-12}$ & $7.11 \times 10^{-11}$ & $5.12 \times 10^{-16}$ & $2.96 \times 10^{-6}$ \\
\hline
\end{tabular}

TABLE III. Numerical values for the coefficients $C_{i}$ defined in Eq. (35) for $B^{-} \rightarrow K^{-} \tilde{\chi}_{1}^{0} \tilde{\chi}_{1}^{0}$ at the various pseudo-SPS points described in the text. The final column shows the ratio of the branching ratio for $B^{-} \rightarrow K^{-} \tilde{\chi}_{1}^{0} \tilde{\chi}_{1}^{0}$ to the current experimental upper bound on the branching ratio for $B^{-} \rightarrow K^{-} \nu \bar{\nu}\left(1.4 \times 10^{-5}\right)$.

\begin{tabular}{|c|c|c|c|c|c|c|}
\hline Pseudo-SPS & $\left|C_{\not ̈ \not}\right|$ & $\left|C_{p}\right|$ & $\left|C_{L}\right|$ & $\left|C_{R}\right|$ & BR & $\mathrm{BR} / \exp$ \\
\hline $1 \mathrm{a}$ & $6.49 \times 10^{-6}$ & $3.77 \times 10^{-9}$ & $1.93 \times 10^{-8}$ & $7.22 \times 10^{-7}$ & $3.35 \times 10^{-10}$ & $2.39 \times 10^{-5}$ \\
\hline 2 & $5.44 \times 10^{-7}$ & $3.69 \times 10^{-9}$ & $4.13 \times 10^{-8}$ & $1.83 \times 10^{-7}$ & $2.48 \times 10^{-12}$ & $1.77 \times 10^{-7}$ \\
\hline 3 & $3.00 \times 10^{-6}$ & $1.87 \times 10^{-9}$ & $2.16 \times 10^{-8}$ & $4.54 \times 10^{-7}$ & $7.19 \times 10^{-11}$ & $5.14 \times 10^{-6}$ \\
\hline 4 & $3.31 \times 10^{-6}$ & $3.22 \times 10^{-9}$ & $5.85 \times 10^{-9}$ & $6.38 \times 10^{-6}$ & $2.53 \times 10^{-10}$ & $1.81 \times 10^{-5}$ \\
\hline 5 & $9.50 \times 10^{-6}$ & $1.49 \times 10^{-9}$ & $2.12 \times 10^{-8}$ & $2.27 \times 10^{-7}$ & $7.14 \times 10^{-10}$ & $5.10 \times 10^{-5}$ \\
\hline
\end{tabular}


diagonal flavor structure of the gauge interactions, but the resulting squarks are not necessarily mass eigenstates. The squark propagator can be expanded according to Ref. [90]:

$$
\begin{aligned}
\left\langle\tilde{q}_{\alpha} \tilde{q}_{\beta}^{*}\right\rangle & =\frac{i}{\left(k^{2} \mathbb{1}-\tilde{m}^{2} \mathbb{1}-\delta m^{2}\right)_{\alpha \beta}} \\
& =\frac{i}{k^{2}-\tilde{m}^{2}} \mathbb{1}_{\alpha \beta}+\frac{i}{\left(k^{2}-\tilde{m}^{2}\right)^{2}} \delta m_{\alpha \beta}^{2}+\mathcal{O}\left(\delta m^{4}\right),
\end{aligned}
$$

where $\mathbb{1}$ is the identity matrix. $\alpha$ and $\beta$ are the indices of the $6 \times 6$ squark mass-squared matrix $\tilde{M}^{2}$ and $\tilde{m}^{2}=$ $\sqrt{\left(\tilde{M}^{2}\right)_{\alpha \alpha}\left(\tilde{M}^{2}\right)_{\beta \beta}}$ is the "average" squark mass squared. The off-diagonal elements of the squared squark mass matrix in the super-CKM basis are given by $\delta m_{\alpha \beta}^{2}$.

To avoid large FCNCs, which would be in contradiction with experimental observations, we shall make the common assumption $\tilde{m}^{2} \gg \delta m_{\alpha \beta}^{2}$ [76]. In this case higher order terms in $\delta m_{\alpha \beta}^{2}$ in Eq. (39), denoted by $\mathcal{O}\left(\delta m^{4}\right)$, can usually be neglected. However, if the linear term in $\delta m_{\alpha \beta}^{2}$ in Eq. (39) vanishes, higher orders in $\delta m^{2}$ need to be taken into account.

In what follows we parametrize the amount of flavor violation with the help of the dimensionless mass insertion parameters [76]:

$$
\left(\delta_{i j}^{d}\right)_{X Y} \equiv \frac{\left(\delta m_{d X Y}^{2}\right)_{i j}}{\tilde{m}^{2}}, \quad i \neq j,
$$

where $\left(\delta m_{d X Y}^{2}\right)_{i j}$ with $X Y=L L, R R, L R$, and $R L$ are the off-diagonal elements of the $6 \times 6$ down squark masssquared matrices, which couple squarks of helicity $X$ to squarks of helicity $Y$.

From the nonobservation of FCNCs beyond the standard model contributions, bounds on the mass insertion parameters, Eq. (40), can be derived [76,91-100]:

$$
\begin{array}{lll}
\left|\left(\delta_{d s}^{d}\right)_{L L / R R}\right| \lesssim \mathcal{O}\left(10^{-2}\right), & \left|\left(\delta_{d s}^{d}\right)_{R L / L R}\right| \lesssim \mathcal{O}\left(10^{-3}\right), \\
\left|\left(\delta_{d b}^{d}\right)_{L L / R R}\right| \lesssim \mathcal{O}\left(10^{-1}\right), & \left|\left(\delta_{d b}^{d}\right)_{R L / L R}\right| \lesssim \mathcal{O}\left(10^{-2}\right), \\
\left|\left(\delta_{s b}^{d}\right)_{L L / R R}\right| \lesssim \mathcal{O}\left(10^{-1}\right), & \left|\left(\delta_{s b}^{d}\right)_{R L / L R}\right| \lesssim \mathcal{O}\left(10^{-2}\right) .
\end{array}
$$

Here an average squark mass of $\tilde{m}=500 \mathrm{GeV}$ is assumed.

The bounds in Eq. (41) were obtained mainly from meson-antimeson mixing. In this case, they scale (at leading order) with $\tilde{m} /(500 \mathrm{GeV})$ as long as the ratio of the squark and gluino masses is fixed, i.e., the bounds get weaker for increased squark mass. Note that in addition to experimental constraints, there exist theoretical constraints on all $\left(\delta_{i j}^{d}\right)_{L R}$ from the requirement of vacuum stability [101]. These bounds are comparable or even stronger than those in Eq. (41). Furthermore, they do not become weaker as the SUSY scale is raised.
The constraints on the mass insertions which connect squarks associated with different helicity are usually much more restrictive than those on the "helicity-conserving" mass insertions. We therefore concentrate in the following on processes where flavor violation is mediated by either only left-handed or only right-handed squarks. We thus consider only contributions from either $\delta_{L L}$ or $\delta_{R R}$.

\section{A. Matrix elements and partial width}

We show in Fig. 8 the tree-level Feynman diagrams which allow for the process $K^{-} \rightarrow \pi^{-} \tilde{\chi}_{1}^{0} \tilde{\chi}_{1}^{0}$ assuming a nearly massless binolike neutralino $\tilde{\chi}_{1}^{0}$. We obtain, after employing Fierz transformations, the following quarklevel matrix elements from the diagrams in Fig. 8:

$$
\begin{aligned}
\mathcal{M}_{L L} & =\frac{e^{2}\left(\delta_{d s}^{d}\right)_{L L}}{36 \cos ^{2} \theta_{W} \tilde{m}^{2}}\left[\bar{d} \gamma^{\mu} P_{L} s\right]\left[\bar{u}\left(k_{1}\right) \gamma_{\mu} \gamma_{5} v\left(k_{2}\right)\right], \\
\mathcal{M}_{R R} & =\frac{-e^{2}\left(\delta_{d s}^{d}\right)_{R R}}{9 \cos ^{2} \theta_{W} \tilde{m}^{2}}\left[\bar{d} \gamma^{\mu} P_{R} s\right]\left[\bar{u}\left(k_{1}\right) \gamma_{\mu} \gamma_{5} v\left(k_{2}\right)\right] .
\end{aligned}
$$

Here $\mathcal{M}_{L L}$ and $\mathcal{M}_{R R}$ are the matrix elements involving only left- and only right-handed squarks, respectively. The quark currents are replaced by their corresponding hadronic matrix elements, which are parametrized by the form factors $f_{+}$and $f_{-}$; see the Appendix for details.

From the squared matrix elements, Eq. (A8), we easily obtain the partial width for $K^{-} \rightarrow \pi^{-} \tilde{\chi}_{1}^{0} \tilde{\chi}_{1}^{0}$. Note that we have to introduce an additional factor of $1 / 2$ because this process involves two identical particles in the final state.

The decays,

$$
B^{-} \rightarrow \pi^{-} / K^{-} \tilde{\chi}_{1}^{0} \tilde{\chi}_{1}^{0},
$$

are described by similar squared matrix elements, which can be obtained from Eq. (A8) by replacing the form factors according to Eq. (A6) and, if considering $B^{-} \rightarrow$ $K^{-} \tilde{\chi}_{1}^{0} \tilde{\chi}_{1}^{0}, p_{\pi}\left(m_{\pi}\right)$ by $p_{K}\left(m_{K}\right)$.

\section{B. Branching ratios and excluded parameter space}

The SM process $K^{-} \rightarrow \pi^{-} \nu \bar{\nu}$ has the same experimental signature as the kaon decay into a pion plus neutralinos, namely, a charged pion and missing energy. Here $\nu$ is a neutrino of arbitrary flavor. The theoretical prediction for the branching ratio for this decay (within the SM) is [71]

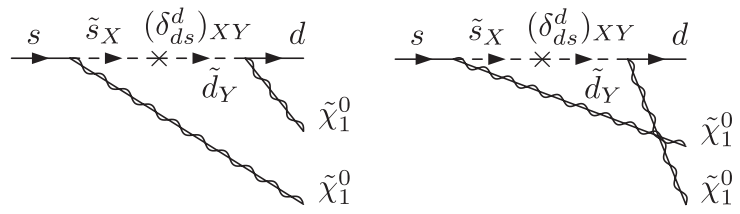

FIG. 8. The tree-level diagrams contributing to the process $K^{-} \rightarrow \pi^{-} \tilde{\chi}_{1}^{0} \tilde{\chi}_{1}^{0}$ without assuming MFV. The FCNCs are mediated by left- $(X, Y=L)$ and right-handed $(X, Y=R)$ squarks, respectively. 


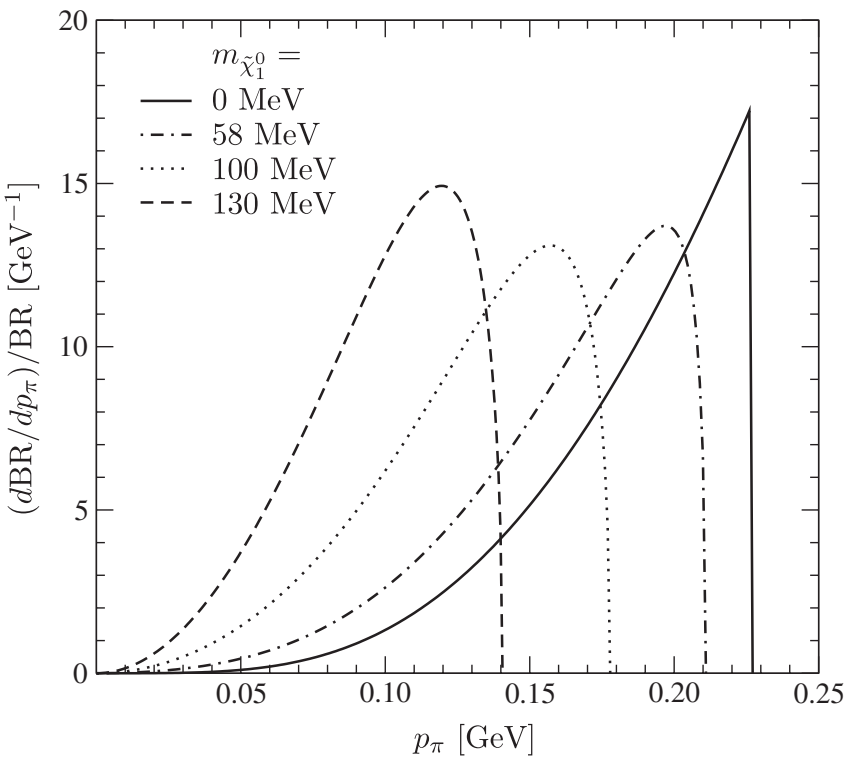

FIG. 9. Pion momentum distribution for the process $K^{-} \rightarrow$ $\pi^{-} \tilde{\chi}_{1}^{0} \tilde{\chi}_{1}^{0}$ for different neutralino masses $m_{\tilde{\chi}_{1}^{0}}$. The distributions are normalized to 1 .

$$
\left.\mathrm{BR}\left(K^{-} \rightarrow \pi^{-} \nu \bar{\nu}\right)\right|_{\text {theory }}=(8.5 \pm 0.7) \times 10^{-11} .
$$

The squared matrix elements of the SM decay depend on the external momenta in exactly the same way as those of the SUSY decay in Eq. (A8) for massless $\tilde{\chi}_{1}^{0}$ 's. The distribution of the pion momentum $p_{\pi}$ for $K^{-} \rightarrow \pi^{-} \tilde{\chi}_{1}^{0} \tilde{\chi}_{1}^{0}$ is then equal to the $p_{\pi}$ distribution of the SM process. However, this is no longer the case for massive $\tilde{\chi}_{1}^{0}$ 's as can be seen in Fig. 9.

We show in Fig. 9 the $p_{\pi}$ distribution $\left(d \mathrm{BR} / d p_{\pi}\right) / \mathrm{BR}$ where $\mathrm{BR}=\operatorname{BR}\left(K^{-} \rightarrow \pi^{-} \tilde{\chi}_{1}^{0} \tilde{\chi}_{1}^{0}\right)$, for different values of $m_{\tilde{\chi}_{1}^{0}}$ in the kaon rest frame. The distributions are normalized to 1 . We have employed the squared matrix element of Eq. (A7) to calculate $\left(d \mathrm{BR} / d p_{\pi}\right) / \mathrm{BR}$. We see that for $m_{\tilde{\chi}_{1}^{0}}>130 \mathrm{MeV}, p_{\pi}$ is smaller than $140 \mathrm{MeV}$. This has important consequences for experimental searches.

The E787 and E949 Collaborations have observed events consistent with the SM decay $K^{-} \rightarrow \pi^{-} \nu \bar{\nu}$. They found that [87]

$$
\left.\operatorname{BR}\left(K^{-} \rightarrow \pi^{-} \nu \bar{\nu}\right)\right|_{\exp }=\left(1.73_{-1.05}^{+1.15}\right) \times 10^{-10},
$$

assuming a $p_{\pi}$ spectrum equal to the SM prediction. To separate the signal from the background, $p_{\pi}$ regions were selected, namely, $211 \mathrm{MeV}<p_{\pi}<229 \mathrm{MeV}$ (region I) [73,102] and $140 \mathrm{MeV}<p_{\pi}<199 \mathrm{MeV}$ (region II) $[87,103]$. These were chosen such that the background $K^{-} \rightarrow \pi^{-} \pi^{0}$ with $p_{\pi} \approx 205 \mathrm{MeV}$ is excluded. It follows from Fig. 9 that the experimental searches in region I (region II) were insensitive to the process $K^{-} \rightarrow$ $\pi^{-} \tilde{\chi}_{1}^{0} \tilde{\chi}_{1}^{0}$ if $m_{\tilde{\chi}_{1}^{0}}>58 \mathrm{MeV}\left(m_{\tilde{\chi}_{1}^{0}}>130 \mathrm{MeV}\right)$, because the respective pion momenta are then too small.
In the following, we will estimate the experimental sensitivity for scenarios with $m_{\tilde{\chi}_{1}^{0}} \neq 0$ with the help of the correction factor

$$
f_{c} \equiv \frac{I\left(m_{\tilde{\chi}_{1}^{0}} \neq 0\right)}{I\left(m_{\tilde{\chi}_{1}^{0}}=0\right)},
$$

with

$$
I\left(m_{\tilde{\chi}_{1}^{0}}\right)=\int_{140 \mathrm{MeV}}^{p_{\pi, \max }}\left(\frac{d \mathrm{BR}}{d p_{\pi}}\right) d p_{\pi},
$$

where $p_{\pi, \max }$ is the maximal kinematically allowed pion momentum for $\tilde{\chi}_{1}^{0}$ 's with mass $m_{\tilde{\chi}_{1}^{0}}$. We set $I\left(m_{\tilde{\chi}_{1}^{0}}\right)$ equal to zero if $p_{\pi, \max }<140 \mathrm{MeV}$.

We show in Fig. 10 the branching ratio for $K^{-} \rightarrow$ $\pi^{-} \tilde{\chi}_{1}^{0} \tilde{\chi}_{1}^{0}$ as a function of $m_{\tilde{\chi}_{1}^{0}}$ and the mass insertion parameter $\left(\delta_{d s}^{d}\right)_{L L}$ (left panel) and $\left(\delta_{d s}^{d}\right)_{R R}$ (right panel). We assume in Eq. (A7) an average squark mass of $\tilde{m}=$ $500 \mathrm{GeV}$. The branching ratios are normalized to the SM prediction, Eq. (44).

The lower solid gray (turquoise) lines in Fig. 10 correspond to regions in the MSSM parameter space where the process $K^{-} \rightarrow \pi^{-} \tilde{\chi}_{1}^{0} \tilde{\chi}_{1}^{0}$ has a branching ratio equal to the SM prediction, Eq. (44). The upper solid gray (turquoise) lines show points in the $m_{\tilde{\chi}_{1}^{0}}-\left(\delta_{d s}^{d}\right)$ planes, where the branching ratio for $K^{-} \rightarrow \pi^{-} \tilde{\chi}_{1}^{0} \tilde{\chi}_{1}^{0}$ is equal to the central experimental value for $\operatorname{BR}\left(K^{-} \rightarrow \pi^{-} \nu \bar{\nu}\right)$ plus $2 \sigma$, Eq. (45), multiplied by the correction factor $f_{c}$, Eq. (46). We assume, as a conservative approach, that the branching ratio for $K^{-} \rightarrow \pi^{-} \nu \bar{\nu}$ is negligible compared to the branching ratio for $K^{-} \rightarrow \pi^{-} \tilde{\chi}_{1}^{0} \tilde{\chi}_{1}^{0}$. With our conservative approach, we can exclude at $2 \sigma$ the MSSM parameter space which lies above the upper solid gray (turquoise) line. As can be seen from Fig. 10, the experiments were unable to probe the region with $m_{\tilde{\chi}_{1}^{0}} \gtrsim 130 \mathrm{MeV}$, although $\operatorname{BR}\left(K^{-} \rightarrow \pi^{-} \tilde{\chi}_{1}^{0} \tilde{\chi}_{1}^{0}\right)$ can be 1 order of magnitude larger then the respective SM process, cf. Eq. (44). Future experiments, such as CERN P-326/NA62 [104], will be able to explore roughly the MSSM parameter space between the two solid gray (turquoise) lines as long as $m_{\tilde{\chi}_{1}^{0}} \lesssim$ $130 \mathrm{MeV}$.

In Ref. [92], bounds on the mass insertion parameters $\left(\delta_{d s}^{d}\right)_{L L / R R}$ were derived from the mass splitting of the $K^{0}-\bar{K}^{0}$ system. For an average squark mass of $\tilde{m}=$ $500 \mathrm{GeV}$, three different bounds on $\left(\delta_{d s}^{d}\right)_{L L / R R}$ were obtained assuming three different ratios $x$ of gluino, $m_{\tilde{g}}$, and squark masses

$$
\begin{aligned}
& x \equiv m_{\tilde{g}}^{2} / \tilde{m}^{2}=4.0 \Rightarrow\left(\delta_{d s}^{d}\right)_{L L / R R} \leq 1.1 \times 10^{-1}, \\
& x \equiv m_{\tilde{g}}^{2} / \tilde{m}^{2}=1.0 \Rightarrow\left(\delta_{d s}^{d}\right)_{L L / R R} \leq 4.6 \times 10^{-2}, \\
& x \equiv m_{\tilde{g}}^{2} / \tilde{m}^{2}=0.3 \Rightarrow\left(\delta_{d s}^{d}\right)_{L L / R R} \leq 2.2 \times 10^{-2} .
\end{aligned}
$$

The horizontal dashed lines in Fig. 10 correspond to the bounds above. Thus, with our calculation of the non-MFV 

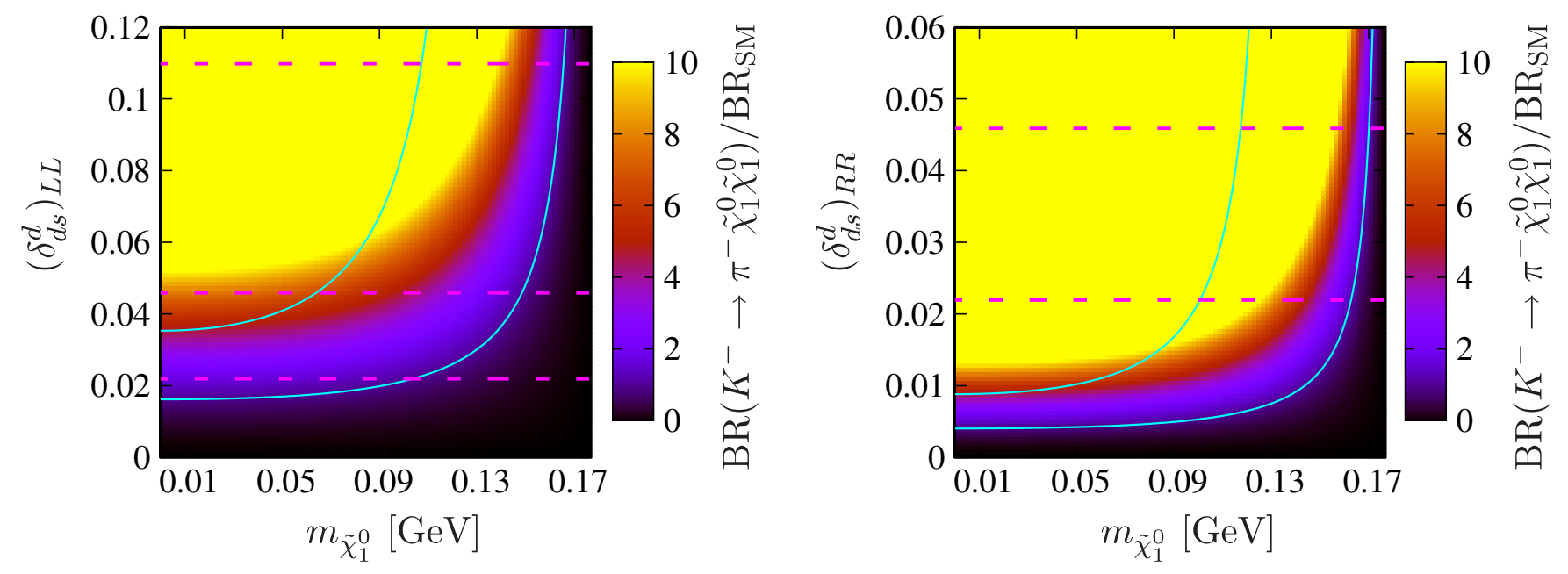

FIG. 10 (color online). Branching ratios for $K^{-} \rightarrow \pi^{-} \tilde{\chi}_{1}^{0} \tilde{\chi}_{1}^{0}$ as a function of the neutralino mass $m_{\tilde{\chi}_{1}^{0}}$ and the mass insertion $\left(\delta_{d s}^{d}\right)_{L L}$ (left panel) and $\left(\delta_{d s}^{d}\right)_{R R}$ (right panel). The branching ratios are normalized to the $\operatorname{SM}$ prediction $\operatorname{BR}\left(K^{\underline{\chi}_{1}} \rightarrow \pi^{-} \nu \bar{\nu}\right)=8.5 \times 10^{-11}[71]$. We assume an average squark mass of $\tilde{m}=500 \mathrm{GeV}$. The lightest gray (yellow) region corresponds to normalized BRs $\geq 10$. The upper (lower) solid gray (turquoise) line corresponds to the experimentally measured branching ratio plus $2 \sigma$, Eq. (45), multiplied with the correction factor $f_{c}$, Eq. (46) (SM prediction) for $K^{-} \rightarrow \pi^{-} \nu \bar{\nu}$. The dashed lines show upper bounds on the mass insertions $\left(\delta_{d s}^{d}\right)_{L L / R R}$ obtained from $K^{0}-\bar{K}^{0}$ mixing [92] for different ratios of squark and gluino masses, cf. Eq. (48).

process $K^{-} \rightarrow \pi^{-} \tilde{\chi}_{1}^{0} \tilde{\chi}_{1}^{0}$, we are able for the first time to exclude the MSSM parameter space which lies above the upper solid gray (turquoise) lines and below one of the dashed lines. Note that the weakest bound on $\left(\delta_{d s}^{d}\right)_{R R}(x=$ 4) lies outside the right panel in Fig. 10.

The plot on the left in Fig. 10 with $\left(\delta_{d s}^{d}\right)_{L L} \neq 0$ looks qualitatively the same as that on the right in Fig. 10 with $\left(\delta_{d s}^{d}\right)_{R R} \neq 0$. The main difference is that the branching ratio for any specific value of $\left(\delta_{d s}^{d}\right)$ and $m_{\tilde{\chi}_{1}^{0}}$ is 16 times larger in the right than in the left panel. This is because the light neutralino has to be binolike and therefore couples to hypercharge. This is larger for right-handed particles than the respective left-handed particles in the MSSM. We see in Fig. 10 that for a massless $\tilde{\chi}_{1}^{0}$, we can strengthen the

$$
\begin{aligned}
x=4.0 & \Rightarrow\left(\delta_{d b}^{d}\right)_{L L / R R} \leq 7.0 \times 10^{-1}, \\
x=1.0 & \Rightarrow\left(\delta_{d b}^{d}\right)_{L L / R R} \leq 1.4 \times 10^{-1}, \\
x=0.3 & \Rightarrow\left(\delta_{s b}^{d}\right)_{L L / R R} \leq 2.3 \times 10^{-1}, \\
x=0.25 & \Rightarrow\left(\delta_{d b}^{d}\right)_{L L / R R} \leq 6.2 \times 10^{-2} .
\end{aligned}
$$

bound on the flavor-violating mass insertion parameter $\left(\delta_{d s}^{d}\right)_{R R}$ by a factor of between 2 and 10 depending on the ratio of squark mass to gluino mass, cf. Eq. (48).

As pointed out in Sec. IVA, we can also employ our squared matrix elements, Eq. (A7), to calculate the branching ratios of the flavor-changing $B$ decays, Eq. (43). Experimentally, so far only upper bounds for these processes exist $[9,74,105]$ :

$$
\begin{aligned}
& \mathrm{BR}\left(B^{-} \rightarrow \pi^{-} \nu \bar{\nu}\right) \leq 1.0 \times 10^{-4}, \\
& \mathrm{BR}\left(B^{-} \rightarrow K^{-} \nu \bar{\nu}\right) \leq 1.4 \times 10^{-5} .
\end{aligned}
$$

The upper bounds on the respective mass insertion parameters are

$$
\begin{aligned}
& \left(\delta_{s b}^{d}\right)_{L L / R R} \leq 1.1 \times 10^{0}, \\
& \left(\delta_{s b}^{d}\right)_{L L / R R} \leq 4.8 \times 10^{-1}
\end{aligned}
$$

bounds in Eq. (50). In addition, SUSY contributions to $B_{s}^{0}-\bar{B}_{s}^{0}$ mixing can be dominated by two-loop double Higgs penguins for large $\tan \beta$. For more details, see, for example, Refs. [76,95,99,100,107] and references therein. However, these bounds are highly model dependent, i.e., they depend not only on the squark and gluino masses. We will therefore not consider them in our analysis.

We show in Fig. 11 the branching ratios for $B^{-} \rightarrow$ $\pi^{-} \tilde{\chi}_{1}^{0} \tilde{\chi}_{1}^{0}$ as a function of $m_{\tilde{\chi}_{1}^{0}}$ and the mass insertions 

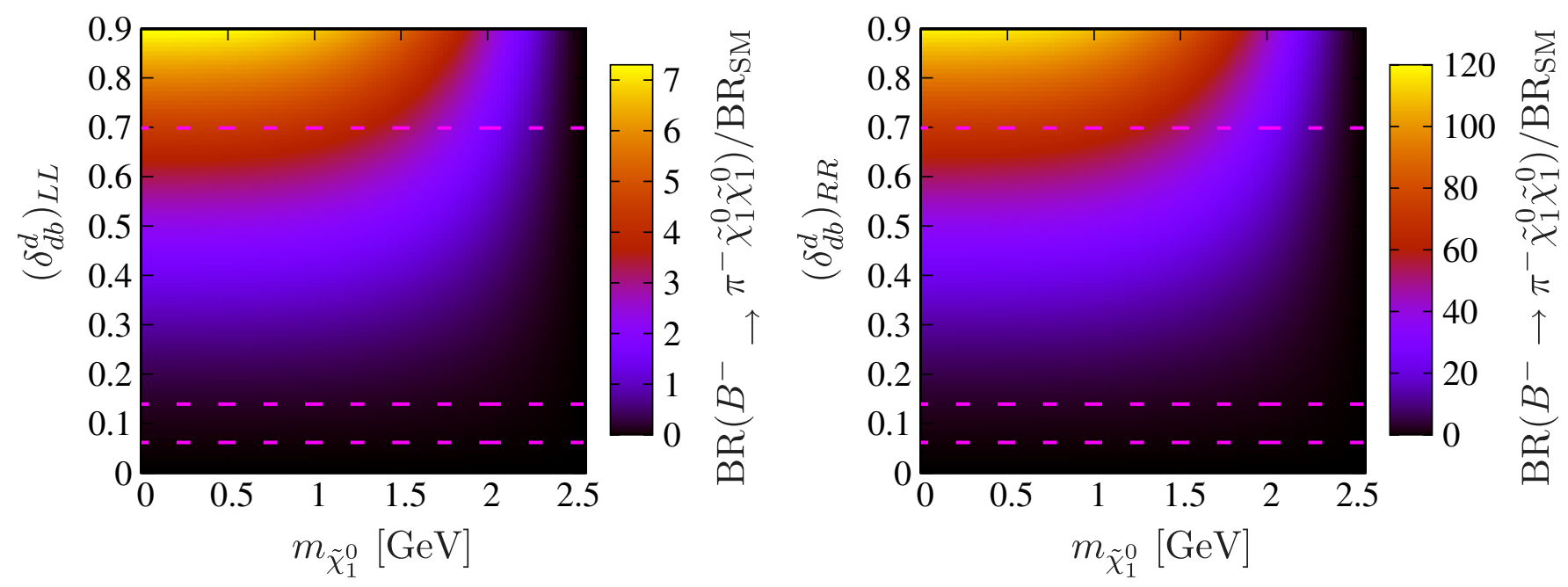

FIG. 11 (color online). Branching ratios for $B^{-} \rightarrow \pi^{-} \tilde{\chi}_{1}^{0} \tilde{\chi}_{1}^{0}$ as a function of the neutralino mass $m_{\tilde{\chi}_{1}^{0}}$ and the mass insertion $\left(\delta_{d b}^{d}\right)_{L L}$ (left panel) and $\left(\delta_{d b}^{d}\right)_{R R}$ (right panel). The branching ratio is normalized to the calculated BR for $B^{-} \stackrel{\chi_{1}}{\rightarrow} \pi^{-} \nu \bar{\nu}$ within the SM, which is $2.2 \times 10^{-7}$ [115]. We assume an average squark mass of $\tilde{m}=500 \mathrm{GeV}$. The dashed lines show upper bounds on the mass insertions $\left(\delta_{d b}^{d}\right)_{L L / R R}$ mainly obtained from $B_{d}^{0}-\bar{B}_{d}^{0}$ mixing for different ratios of squark mass to gluino mass [94], cf. Eq. (50).

$\left(\delta_{d b}^{d}\right)_{L L}$ (left panel) and $\left(\delta_{d b}^{d}\right)_{R R}$ (right panel). The dashed lines correspond to the upper bounds on the mass insertions as given in Eq. (50). The allowed region for nonvanishing $\left(\delta_{d b}^{d}\right)_{L L}\left[\left(\delta_{d b}^{d}\right)_{R R}\right]$, i.e., the region below the dashed lines, lies at least 2 (1) orders of magnitude below the current experimental upper bound of $1.0 \times 10^{-4}$, cf. Eq. (49). We can thus not exclude additional regions of the MSSM parameter space from the upper bound on the branching ratio. However, a future super $B$ factory will be able to explore large parts of the parameter space shown in Fig. 11
[108], i.e., branching ratios down to $\mathcal{O}\left(10^{-6}-10^{-7}\right)$. Note that if $m_{\tilde{\chi}_{1}^{0}} \gtrsim 1 \mathrm{GeV}$, the momentum of the pion or kaon from the $B$ meson decay may be too soft to pass experimental cuts.

Finally we show in Fig. 12 the branching ratios for the process $B^{-} \rightarrow K^{-} \tilde{\chi}_{1}^{0} \tilde{\chi}_{1}^{0}$ as a function of $m_{\tilde{\chi}_{1}^{0}}$ and $\left(\delta_{s b}^{d}\right)_{L L / R R}$. The numerical values are of the same order of magnitude as those in Fig. 11. Differences are mainly due to different form factors, see Eq. (A6). In contrast to the $B$ decay into a pion, the decay into a kaon has a stricter
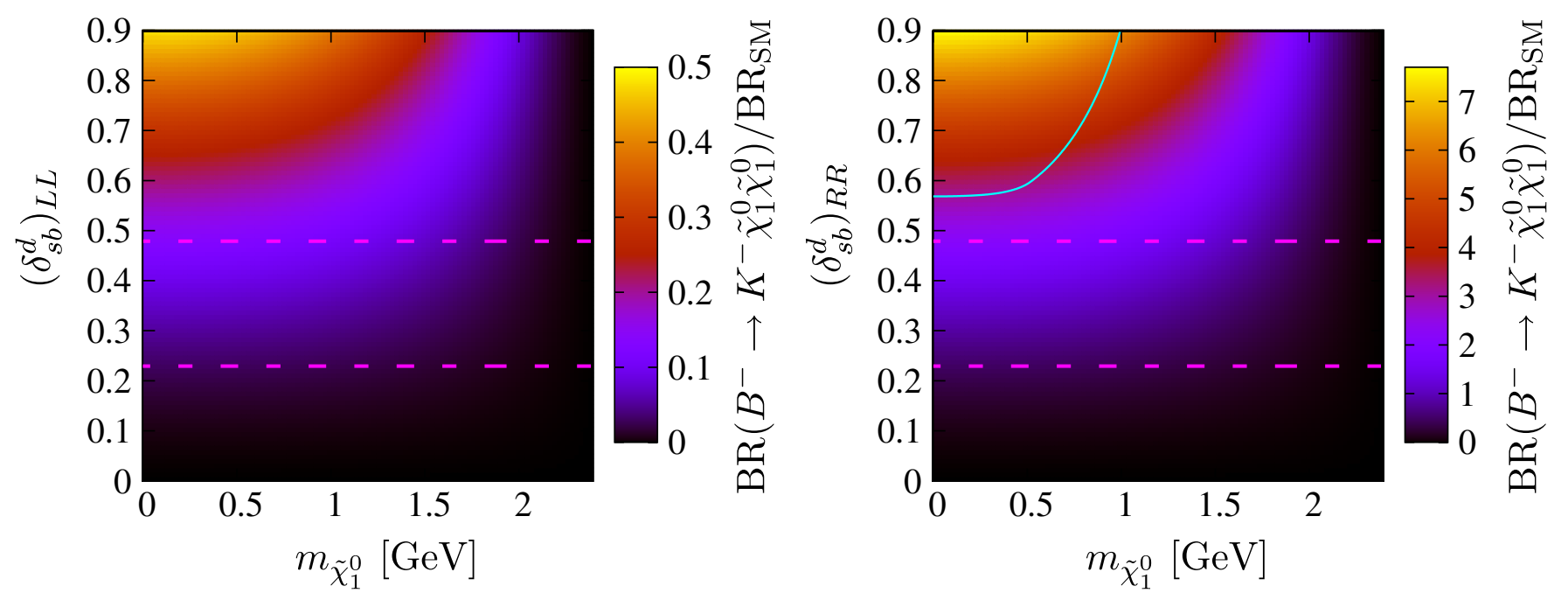

FIG. 12 (color online). Branching ratios for $B^{-} \rightarrow K^{-} \tilde{\chi}_{1}^{0} \tilde{\chi}_{1}^{0}$ as a function of the neutralino mass $m_{\tilde{\chi}_{1}^{0}}$ and the mass insertion $\left(\delta_{s b}^{d}\right)_{L L}$ (left panel) and $\left(\delta_{s b}^{d}\right)_{R R}$ (right panel). The branching ratio is normalized to the calculated BR for $B^{-} \stackrel{\chi_{1}}{\rightarrow} K^{-} \nu \bar{\nu}$ within the SM, which is $4.5 \times 10^{-6}[116,117]$. We assume an average squark mass of $\tilde{m}=500 \mathrm{GeV}$. The solid gray (turquoise) line corresponds to the experimental upper bound, Eq. (49), multiplied by a correction factor analogously to Eq. (46). The dashed lines show upper bounds on the mass insertions $\left(\delta_{s b}^{d}\right)_{L L / R R}$ obtained from $B_{s}^{0}-\bar{B}_{s}^{0}$ mixing for different ratios of squark mass to gluino mass, cf. Eq. (50). The weakest bound on $\left(\delta_{s b}^{d}\right)(x=4)$ lies outside the figure. 
upper bound on the respective branching ratio, namely, $1.4 \times 10^{-5}$, cf. Eq. (49). The solid gray (turquoise) line in Fig. 12 corresponds to this upper bound multiplied by a correction factor analogous to Eq. (46). We have taken into account that the BELLE Collaboration required the kaon momentum to lie within a window of 1.6 and $2.5 \mathrm{GeV}$ [74]. We can therefore exclude the MSSM parameter space above the gray (turquoise) line. Again, with a future super $B$ factory we will be able to explore large regions of the parameter space in Fig. 12 [108].

To conclude this section, let us briefly comment on the validity of the mass insertion approximation, Eq. (39). For the decay $K^{-} \rightarrow \pi^{-} \tilde{\chi}_{1}^{0} \tilde{\chi}_{1}^{0}$, the relevant mass insertion parameters are of the order $\delta_{d s}^{d}=\mathcal{O}\left(10^{-2}\right)$ and an expansion in $\delta_{d s}^{d}$ converges very fast. For the $B$ decays, however, we have considered mass insertions of up to $\delta_{d b / s b}^{d}=0.9$, cf. Figs. 11 and 12. While it is clear that the analysis should eventually be refined by taking into account higher order terms in the expansion Eq. (39), our approximation is sufficient to demonstrate that $B$ decays into very light $\tilde{\chi}_{1}^{0}$ can be tested with the next generation of $B$ factories.

\section{LIGHT NEUTRALINO PRODUCTION AT HADRON COLLIDERS}

A massless or very light $\tilde{\chi}_{1}^{0}$ that is purely bino is not fundamentally different in terms of collider signals than scenarios where it is $\mathcal{O}(100 \mathrm{GeV})$ and mainly bino, such as SPS1a (97 GeV, 98.5\% bino). The main effect is that the phase space is bigger, in general increasing cross sections, especially where the energy is not much greater than the creation threshold. There are many signals of interest at colliders, not least those involving decay chains, but the masslessness of the lightest neutralino does not fundamentally change those analyses.

It is reasonable to ask whether the increase due to the greater phase space is in conflict with searches at colliders so far. The bino component does not couple to the $Z$ boson at tree level and so constraints from the invisible width of the $Z$ are only applicable to the very small Higgsino components and also to the bino component at the oneloop level. No bounds on the neutralino mass can thus be deduced from the $Z$ width [16,18,20]. Direct searches at past and future $e^{+} e^{-}$colliders have been studied in detail in Refs. [20,27,28,30,32,109]. While no bounds on the mass of the lightest neutralino can be obtained from past searches at LEP and $B$ factories, measurements at a future $e^{+} e^{-}$linear collider might be able to discover light neutralinos through radiative production, $e^{+} e^{-} \rightarrow \tilde{\chi}_{1}^{0} \tilde{\chi}_{1}^{0}+\gamma$.

Direct hadroproduction of light neutralinos in association with a jet,

$$
p \bar{p} / p p \rightarrow \tilde{\chi}_{1}^{0} \tilde{\chi}_{1}^{0}+\text { jet, }
$$

will lead to a spectacular monojet signature. We have calculated the tree-level cross section for the pair production of massless $\tilde{\chi}_{1}^{0}$ plus one jet, Eq. (51), for both the Tevatron $(\sqrt{s}=1.96 \mathrm{TeV})$ and the LHC $(\sqrt{s}=14 \mathrm{TeV})$ using MADGRAPH [110]. We require a jet with transverse momentum of at least $80 \mathrm{GeV}$. The numerical results are collected in Table IV for the pseudo-SPS points described in Sec. III and are compared to the original SPS points with $\tilde{\chi}_{1}^{0}$ masses of typically $\mathcal{O}(100 \mathrm{GeV})$. Even though the Tevatron cross sections for massless $\tilde{\chi}_{1}^{0}$ are enhanced by a factor up to 2.5 with respect to the original SPS cross sections with massive neutralinos, the expected number of events is only about 2 or less for the full $6 \mathrm{fb}^{-1}$ of integrated luminosity of run II so far. Given the large SM backgrounds, such as $p \bar{p} \rightarrow Z(\rightarrow \nu \bar{\nu})+$ jet, we conclude that current and future monojet searches at the Tevatron $[111,112]$ will not be sensitive to the direct pair production of light neutralinos. Similar conclusions hold for radiative production with an additional photon, $p \bar{p} \rightarrow \tilde{\chi}_{1}^{0} \tilde{\chi}_{1}^{0}+\gamma$.

At the LHC, light neutralino pair production with jets is only enhanced from the massive case by about $20 \%$. Still, with cross sections of $\mathcal{O}(100 \mathrm{fb})$, see Table IV, detection of these processes should be possible with sufficiently high luminosity and an excellent understanding of SM backgrounds [113,114].

\section{CONCLUSION}

Rare meson decays provide a sensitive test for the presence of new physics. In this paper we have studied the decay of pseudoscalar and vector mesons to light neutralinos in the MSSM. We have presented details of the calculations and explicit formulas for the two-body decays $M \rightarrow \tilde{\chi}_{1}^{0} \tilde{\chi}_{1}^{0}$, where $M=\pi^{0}, \eta, \eta^{\prime}, \rho^{0}, \omega, \phi$, $J / \psi$, and $Y$. Furthermore, we have performed the first

TABLE IV. Monojet cross sections $\sigma\left(p \bar{p} / p p \rightarrow \tilde{\chi}_{1}^{0} \tilde{\chi}_{1}^{0}+\right.$ jet $)$ at the Tevatron and at the LHC. Shown are results for the pseudo-SPS points with massless $\tilde{\chi}_{1}^{0}$ in comparison to the original SPS cross sections with massive neutralinos.

\begin{tabular}{lcccc}
\hline \hline & \multicolumn{2}{c}{ Tevatron cross section } & \multicolumn{2}{c}{ LHC cross section } \\
(Pseudo-)SPS & Pseudo-SPS & Normal SPS & Pseudo-SPS & Normal SPS \\
\hline $1 \mathrm{a}$ & $2.96 \times 10^{-1} \mathrm{fb}$ & $1.68 \times 10^{-1} \mathrm{fb}$ & $3.26 \times 10^{+2} \mathrm{fb}$ & $2.73 \times 10^{+2} \mathrm{fb}$ \\
2 & $2.88 \times 10^{-3} \mathrm{fb}$ & $1.99 \times 10^{-3} \mathrm{fb}$ & $3.25 \times 10^{+2} \mathrm{fb}$ & $3.01 \times 10^{+2} \mathrm{fb}$ \\
3 & $2.66 \times 10^{-2} \mathrm{fb}$ & $7.75 \times 10^{-3} \mathrm{fb}$ & $5.52 \times 10^{+1} \mathrm{fb}$ & $4.61 \times 10^{+1} \mathrm{fb}$ \\
4 & $4.48 \times 10^{-2} \mathrm{fb}$ & $1.84 \times 10^{-2} \mathrm{fb}$ & $8.59 \times 10^{+1} \mathrm{fb}$ & $7.14 \times 10^{+1} \mathrm{fb}$ \\
5 & $9.18 \times 10^{-2} \mathrm{fb}$ & $3.78 \times 10^{-2} \mathrm{fb}$ & $1.54 \times 10^{+2} \mathrm{fb}$ & $1.32 \times 10^{+2} \mathrm{fb}$ \\
\hline \hline
\end{tabular}


complete calculation of the loop-induced decays $K^{-} \rightarrow$ $\pi^{-} \tilde{\chi}_{1}^{0} \tilde{\chi}_{1}^{0}$ and $B^{-} \rightarrow K^{-} \tilde{\chi}_{1}^{0} \tilde{\chi}_{1}^{0}$. Considering various MSSM scenarios we find that the supersymmetric branching ratios are several orders of magnitude smaller then the SM processes with neutrinos instead of neutralinos in the final state. Consequently, no bounds on the neutralino mass can be inferred from rare meson decays in the MSSM with minimal flavor violation. However, the branching ratios for the $K^{-} \rightarrow \pi^{-} \tilde{\chi}_{1}^{0} \tilde{\chi}_{1}^{0}$ and $B^{-} \rightarrow K^{-} \tilde{\chi}_{1}^{0} \tilde{\chi}_{1}^{0}$ decays may be significantly enhanced when one allows for nonminimal flavor violation. We find new constraints on the MSSM parameter space for such scenarios and argue that future experiments in the kaon and $B$ meson sector may be able probe light neutralinos with masses up to approximately $1 \mathrm{GeV}$ from rare decays. We have also considered searches for light neutralinos from monojet signatures at hadron colliders. While current and future monojet searches at the Tevatron will not be sensitive to the direct pair production of light neutralinos, the detection of these processes should be possible at the LHC with sufficiently high luminosity and an excellent understanding of SM backgrounds.

\section{ACKNOWLEDGMENTS}

We want to thank JoAnne Hewett, Gudrun Hiller, Olaf Kittel, Christopher Smith, and Bryan Webber for discussions and suggestions. This work is supported in part by the European Community's Marie-Curie Research Training Network under Contract No. MRTN-CT-2006-035505 "Tools and Precision Calculations for Physics Discoveries at Colliders", the DFG SFB/TR9 "Computational Particle Physics," and the Helmholtz Alliance "Physics at the Terascale." S. G. thanks the Deutsche Telekom Stiftung and the Bonn-Cologne Graduate School of Physics and Astronomy for financial support. D. K. thanks the Queen Mary, University of London graduate school for financial support.

\section{APPENDIX: FORM FACTORS FOR $K^{-} \rightarrow \pi^{-} \tilde{\chi}_{1}^{0} \tilde{\chi}_{1}^{0}$ AND $B^{-} \rightarrow K^{-} / \pi^{-} \tilde{\boldsymbol{\chi}}_{1}^{0} \tilde{\boldsymbol{\chi}}_{\mathbf{1}}^{0}$}

For the decay $K^{-} \rightarrow \pi^{-} \tilde{\chi}_{1}^{0} \tilde{\chi}_{1}^{0}$, the quark currents need to be replaced by their corresponding hadronic matrix elements involving a kaon $K^{-}$and a pion $\pi^{-}$[9]:

$$
\begin{aligned}
& \left\langle\pi^{-}\left(p_{\pi}\right)\left|\bar{d} \gamma^{\mu} P_{L / R} s\right| K^{-}\left(p_{K}\right)\right\rangle \\
& \quad=\frac{1}{2}\left[f_{+}(t)\left(p_{K}+p_{\pi}\right)^{\mu}+f_{-}(t)\left(p_{K}-p_{\pi}\right)^{\mu}\right],
\end{aligned}
$$

and

$$
f_{-}(t)=\left[f_{0}(t)-f_{+}(t)\right] \frac{\left(m_{k}^{2}-m_{\pi}^{2}\right)}{t},
$$

where $t$ is defined via $t=\left(p_{K}-p_{\pi}\right)^{2}$. The functions $f_{+}$ and $f_{0}$ are the so-called $K_{l 3}$ form factors. We describe them with the help of the linear approximation [9]

$$
f_{+/ 0}(t)=f_{+/ 0}(0)\left[1+\lambda_{+/ 0} \frac{t}{m_{\pi}^{2}}\right]
$$

with $\lambda_{+}=2.96 \times 10^{-2}, \lambda_{0}=1.96 \times 10^{-2}$, and $f_{+}(0)=$ $f_{0}(0)=1.013$, where we calculated $f_{+}(0)$ using the measured branching ratio for $K^{-} \rightarrow \pi^{0} e^{-} \bar{\nu}_{e}$ and isospin symmetry, i.e.,

$$
\begin{aligned}
& \left\langle\pi^{-}\left(p_{\pi}\right)\left|\bar{d} \gamma^{\mu} P_{L / R} s\right| K^{-}\left(p_{K}\right)\right\rangle \\
& \quad=\sqrt{2}\left\langle\pi^{0}\left(p_{\pi}\right)\left|\bar{u} \gamma^{\mu} P_{L / R} s\right| K^{-}\left(p_{K}\right)\right\rangle .
\end{aligned}
$$

The decays

$$
B^{-} \rightarrow \pi^{-} / K^{-} \tilde{\chi}_{1}^{0} \tilde{\chi}_{1}^{0}
$$

can be described in a similar way. One has to replace in Eq. (A1) $p_{K}$ by $p_{B}$ and, if one is considering $B^{-} \rightarrow$ $K^{-} \tilde{\chi}_{1}^{0} \tilde{\chi}_{1}^{0}, p_{\pi}$ by $p_{K}$. For the parametrization of the respective form factors $f_{+}^{\pi / K}$ and $f_{0}^{\pi / K}$, we use $[85,86]$

$$
\begin{aligned}
f_{+}^{\pi} & =\frac{r_{1}^{\pi}}{1-t /\left(m_{1}^{\pi}\right)^{2}}+\frac{r_{2}^{\pi}}{1-t /\left(m_{2}^{\pi}\right)^{2}}, \\
f_{+}^{K} & =\frac{r_{1}^{K}}{1-t /\left(m_{1}^{K}\right)^{2}}+\frac{r_{2}^{K}}{\left[1-t /\left(m_{1}^{K}\right)^{2}\right]^{2}}, \\
f_{0}^{\pi / K} & =\frac{r_{3}^{\pi / K}}{1-t /\left(m_{3}^{\pi / K}\right)^{2}},
\end{aligned}
$$

where $m_{1}^{\pi}=5.32 \mathrm{GeV}$ and $m_{1}^{K}=5.41 \mathrm{GeV}$ are the masses of the $B^{*}$ and $B_{s}^{*}$ vector mesons, respectively. The fit parameters are $r_{1}^{\pi}=0.744, r_{2}^{\pi}=-0.486,\left(m_{2}^{\pi}\right)^{2}=$ $40.73 \mathrm{GeV}^{2}, r_{1}^{K}=0.162, r_{2}^{K}=0.173, r_{3}^{\pi}=0.258, r_{3}^{K}=$ $0.330,\left(m_{3}^{\pi}\right)^{2}=33.81 \mathrm{GeV}^{2}$, and $\left(m_{3}^{K}\right)^{2}=37.46 \mathrm{GeV}^{2}$.

Using this parametrization for the non-MFV $\mathrm{K}^{-} \rightarrow$ $\pi^{-} \tilde{\chi}_{1}^{0} \tilde{\chi}_{1}^{0}$ decay, we obtain for the squared matrix elements summed over final-state spins

$$
\begin{aligned}
\overline{\left|\mathcal{M}_{L L / R R}\right|^{2}}= & C_{L L / R R} \frac{e^{4}\left(\delta_{d s}^{d}\right)_{L L / R R}^{2}}{\cos ^{4} \theta_{W} \tilde{m}^{4}} \\
& \times\left(\overline{\mathcal{M}_{++}^{2}}+\overline{\mathcal{M}_{+-}^{2}}+\overline{\mathcal{M}_{--}^{2}}\right),
\end{aligned}
$$

with

$$
\begin{aligned}
\overline{\mathcal{M}_{++}^{2}}= & 4 f_{+}^{2}\left[m_{\tilde{\chi}_{1}^{0}}^{2}\left(m_{\tilde{\chi}_{1}^{0}}^{2}+m_{\pi}^{2}\right)+\left(k_{1} \cdot k_{2}\right)\left(m_{\tilde{\chi}_{1}^{0}}^{2}-m_{\pi}^{2}\right)\right. \\
& +2 m_{\tilde{\chi}_{1}^{0}}^{2}\left(k_{1} \cdot p_{\pi}\right)+2 m_{\tilde{\chi}_{1}^{0}}^{2}\left(k_{2} \cdot p_{\pi}\right) \\
& \left.+2\left(k_{1} \cdot p_{\pi}\right)\left(k_{2} \cdot p_{\pi}\right)\right], \\
\overline{\mathcal{M}_{+-}^{2}}= & 8 f_{+} f_{-} m_{\tilde{\chi}_{1}^{0}}^{2}\left[m_{\tilde{\chi}_{1}^{0}}^{2}+\left(k_{1} \cdot k_{2}\right)+\left(k_{1} \cdot p_{\pi}\right)\right. \\
& \left.+\left(k_{2} \cdot p_{\pi}\right)\right], \\
\overline{\mathcal{M}_{--}^{2}}= & 4 f_{-}^{2}\left[m_{\tilde{\chi}_{1}^{0}}^{2}\left(k_{1} \cdot k_{2}\right)+m_{\tilde{\chi}_{1}^{0}}^{4}\right]
\end{aligned}
$$

and the constants $C_{L L}=1 / 1296$ and $C_{R R}=1 / 81$. The large difference between $C_{L L}$ and $C_{R R}$ originates from the different hypercharges of right- and left-handed squarks, with which they couple to the binolike $\tilde{\chi}_{1}^{0}$. 
[1] Yu. A. Golfand and E. P. Likhtman, JETP Lett. 13, 452 (1971); J. Wess and B. Zumino, Nucl. Phys. B70, 39 (1974).

[2] H. P. Nilles, Phys. Rep. 110, 1 (1984).

[3] H. E. Haber and G. L. Kane, Phys. Rep. 117, 75 (1985).

[4] H. K. Dreiner, C. Luhn, and M. Thormeier, Phys. Rev. D 73, 075007 (2006).

[5] G. R. Farrar and P. Fayet, Phys. Lett. 76B, 575 (1978).

[6] J. R. Ellis, J. S. Hagelin, D. V. Nanopoulos, K. A. Olive, and M. Srednicki, Nucl. Phys. B238, 453 (1984).

[7] H. Pagels and J.R. Primack, Phys. Rev. Lett. 48, 223 (1982).

[8] H. Goldberg, Phys. Rev. Lett. 50, 1419 (1983).

[9] C. Amsler et al. (Particle Data Group), Phys. Lett. B 667, 1 (2008).

[10] J. Abdallah et al. (DELPHI Collaboration), Eur. Phys. J. C 31, 421 (2003).

[11] LEP Higgs Working Group, LHWG Note 2005-01, 2005.

[12] A. Bartl, H. Fraas, W. Majerotto, and N. Oshimo, Phys. Rev. D 40, 1594 (1989).

[13] I. Gogoladze, J. D. Lykken, C. Macesanu, and S. Nandi, Phys. Rev. D 68, 073004 (2003).

[14] H. K. Dreiner, C. Hanhart, U. Langenfeld, and D. R. Phillips, Phys. Rev. D 68, 055004 (2003).

[15] V. Barger, P. Langacker, and H. S. Lee, Phys. Lett. B 630, 85 (2005).

[16] H. K. Dreiner, S. Heinemeyer, O. Kittel, U. Langenfeld, A. M. Weber, and G. Weiglein, in Proceedings of the 2007 International Linear Collider Workshop (LCWSO7 and ILC07), Hamburg, Germany, 2007 (DESY, Hamburg, Germany, 2007), pp. SUS06.

[17] T. Fritzsche and W. Hollik, Eur. Phys. J. C 24, 619 (2002).

[18] H. K. Dreiner, S. Heinemeyer, O. Kittel, U. Langenfeld, A. M. Weber, and G. Weiglein, arXiv:0901.3485.

[19] J. R. Ellis, K. A. Olive, S. Sarkar, and D. W. Sciama, Phys. Lett. B 215, 404 (1988).

[20] D. Choudhury, H. K. Dreiner, P. Richardson, and S. Sarkar, Phys. Rev. D 61, 095009 (2000).

[21] M. Kachelriess, J. High Energy Phys. 02 (2000) 010.

[22] D. Hooper and T. Plehn, Phys. Lett. B 562, 18 (2003).

[23] A. Bottino, F. Donato, N. Fornengo, and S. Scopel, Phys. Rev. D 68, 043506 (2003).

[24] G. Belanger, F. Boudjema, A. Cottrant, A. Pukhov, and S. Rosier-Lees, J. High Energy Phys. 03 (2004) 012.

[25] J. F. Gunion, D. Hooper, and B. McElrath, Phys. Rev. D 73, 015011 (2006).

[26] S. Profumo, Phys. Rev. D 78, 023507 (2008).

[27] P. Fayet, Phys. Lett. 117B, 460 (1982).

[28] J. R. Ellis and J. S. Hagelin, Phys. Lett. 122B, 303 (1983).

[29] K. Grassie and P. N. Pandita, Phys. Rev. D 30, 22 (1984).

[30] H. K. Dreiner, O. Kittel, and U. Langenfeld, Phys. Rev. D 74, 115010 (2006).

[31] V. Barger, P. Langacker, I. Lewis, M. McCaskey, G. Shaughnessy, and B. Yencho, Phys. Rev. D 75, 115002 (2007).

[32] H. K. Dreiner, O. Kittel, and U. Langenfeld, Eur. Phys. J. C 54, 277 (2008).

[33] P. H. Chankowski, A. Dabelstein, W. Hollik, W. M. Mosle, S. Pokorski, and J. Rosiek, Nucl. Phys. B417, 101 (1994).

[34] M. K. Gaillard, Y. C. Kao, I. H. Lee, and M. Suzuki, Phys. Lett. 123B, 241 (1983).
[35] J. R. Ellis and J. S. Hagelin, Nucl. Phys. B217, 189 (1983).

[36] T. Kobayashi and M. Kuroda, Phys. Lett. 139B, 208 (1984).

[37] J. F. Nieves and P. B. Pal, Phys. Rev. D 32, 1849 (1985).

[38] M.I. Dobroliubov, A. Y. Ignatiev, and V. A. Matveev, Phys. Lett. B 192, 135 (1987); Sov. J. Nucl. Phys. 47, 296 (1988).

[39] E. Gabrielli and G. F. Giudice, Nucl. Phys. B433, 3 (1995); B507, 549(E) (1997).

[40] V. Silveira and A. Zee, Phys. Lett. 161B, 136 (1985).

[41] J. McDonald, Phys. Rev. D 50, 3637 (1994).

[42] C. P. Burgess, M. Pospelov, and T. ter Veldhuis, Nucl. Phys. B619, 709 (2001).

[43] C. Bird, P. Jackson, R. V. Kowalewski, and M. Pospelov, Phys. Rev. Lett. 93, 201803 (2004).

[44] C. Bird, R. V. Kowalewski, and M. Pospelov, Mod. Phys. Lett. A 21, 457 (2006).

[45] C. Boehm, T. A. Ensslin, and J. Silk, J. Phys. G 30, 279 (2004).

[46] C. Boehm and P. Fayet, Nucl. Phys. B683, 219 (2004).

[47] B. McElrath, Phys. Rev. D 72, 103508 (2005).

[48] P. Fayet, Phys. Rev. D 74, 054034 (2006).

[49] P. Fayet, Phys. Rev. D 75, 115017 (2007).

[50] P. Fayet, Phys. Rev. D 70, 023514 (2004).

[51] P. Fayet, arXiv:hep-ph/0408357.

[52] G. Hiller, Phys. Rev. D 70, 034018 (2004).

[53] J.L. Feng and J. Kumar, Phys. Rev. Lett. 101, 231301 (2008).

[54] D. McKeen, Phys. Rev. D 79, 114001 (2009).

[55] A. V. Artamonov et al. (E949 Collaboration), Phys. Rev. D 72, 091102 (2005).

[56] J. Rich and D. R. Winn, Phys. Rev. D 14, 1283 (1976).

[57] J. R. Ellis and S. Rudaz, Phys. Lett. 128B, 248 (1983).

[58] B. A. Campbell, J. A. Scott, and M. K. Sundaresan, Phys. Lett. 131B, 213 (1983).

[59] R. Adhikari and B. Mukhopadhyaya, Phys. Rev. D 52, 3125 (1995).

[60] R. Adhikari and B. Mukhopadhyaya, Phys. Lett. B 353, 228 (1995).

[61] L. N. Chang, O. Lebedev, and J. N. Ng, Phys. Lett. B 441, 419 (1998).

[62] D. Bailin and A. Love, Graduate Student Series in Physics (IOP, Bristol, UK, 1994) p. 322.

[63] H. K. Dreiner, H.E. Haber, and S.P. Martin, arXiv:0812.1594.

[64] W. M. Yao et al. (Particle Data Group), J. Phys. G 33, 1 (2006).

[65] H. Leutwyler, Nucl. Phys. B, Proc. Suppl. 64, 223 (1998).

[66] T. Feldmann, P. Kroll, and B. Stech, Phys. Rev. D 58, 114006 (1998).

[67] H. K. Dreiner, M. Krämer, and B. O’Leary, Phys. Rev. D 75, 114016 (2007).

[68] N. Brambilla, M. Krämer, R. Mussa, A. Vairo, et al. (Quarkonium Working Group), arXiv:hep-ph/0412158.

[69] M. Ablikim et al. (BES Collaboration), Phys. Rev. Lett. 100, 192001 (2008).

[70] O. Tajima et al. (Belle Collaboration), Phys. Rev. Lett. 98, 132001 (2007).

[71] G. Isidori, F. Mescia, and C. Smith, Nucl. Phys. B718, 319 (2005); A. J. Buras, M. Gorbahn, U. Haisch, and U. Nierste, Phys. Rev. Lett. 95, 261805 (2005); J. High 
Energy Phys. 11 (2006) 002; F. Mescia and C. Smith, Phys. Rev. D 76, 034017 (2007); J. Brod and M. Gorbahn, Phys. Rev. D 78, 034006 (2008).

[72] V. V. Anisimovsky et al. (E949 Collaboration), Phys. Rev. Lett. 93, 031801 (2004).

[73] S. Adler et al. (The E949 Collaboration and E787 Collaboration), Phys. Rev. D 77, 052003 (2008).

[74] K. F. Chen et al. (Belle Collaboration), Phys. Rev. Lett. 99, 221802 (2007).

[75] A. J. Buras, Acta Phys. Pol. B 34, 5615 (2003).

[76] S. Jäger, Eur. Phys. J. C 59, 497 (2009).

[77] B. C. Allanach et al., in Proceedings of the APS/DPF/DPB Summer Study on the Future of Particle Physics (Snowmass 2001), edited by N. Graf [Eur. Phys. J. C 25, 113 (2002)].

[78] B. C. Allanach, Comput. Phys. Commun. 143, 305 (2002).

[79] S. Dimopoulos and H. Georgi, Nucl. Phys. B193, 150 (1981); R. Barbieri and R. Gatto, Phys. Lett. 110B, 211 (1982); J. R. Ellis and D. V. Nanopoulos, Phys. Lett. 110B, 44 (1982).

[80] A. J. Buras, arXiv:hep-ph/9806471.

[81] T. Hahn and M. Perez-Victoria, Comput. Phys. Commun. 118, 153 (1999).

[82] T. Hahn and J. I. Illana, Nucl. Phys. B, Proc. Suppl. 160, 101 (2006).

[83] T. Inami and C. S. Lim, Prog. Theor. Phys. 65, 297 (1981); 65, 1772(E) (1981).

[84] S. I. Nam and H. C. Kim, Phys. Rev. D 75, 094011 (2007).

[85] P. Ball and R. Zwicky, arXiv:hep-ph/0406261.

[86] P. Ball and R. Zwicky, Phys. Rev. D 71, 014015 (2005).

[87] A. V. Artamonov et al. (E949 Collaboration), Phys. Rev. D 79, 092004 (2009).

[88] L. J. Hall, V. A. Kostelecky, and S. Raby, Nucl. Phys. B267, 415 (1986).

[89] M. Dugan, B. Grinstein, and L. J. Hall, Nucl. Phys. B255, 413 (1985).

[90] P.H. Chankowski, O. Lebedev, and S. Pokorski, Nucl. Phys. B717, 190 (2005).

[91] F. Gabbiani, E. Gabrielli, A. Masiero, and L. Silvestrini, Nucl. Phys. B477, 321 (1996).

[92] M. Ciuchini et al., J. High Energy Phys. 10 (1998) 008.

[93] M. Misiak, S. Pokorski, and J. Rosiek, Adv. Ser. Dir. High Energy Phys. 15, 795 (1998).

[94] D. Becirevic et al., Nucl. Phys. B634, 105 (2002).
[95] J. Foster, K. i. Okumura, and L. Roszkowski, Phys. Lett. B 641, 452 (2006).

[96] P. Ball and R. Fleischer, Eur. Phys. J. C 48, 413 (2006).

[97] M. Ciuchini and L. Silvestrini, Phys. Rev. Lett. 97, 021803 (2006).

[98] F. Borzumati, C. Greub, T. Hurth, and D. Wyler, Phys. Rev. D 62, 075005 (2000).

[99] M. Ciuchini, E. Franco, A. Masiero, and L. Silvestrini, Phys. Rev. D 67, 075016 (2003); 68, 079901(E) (2003).

[100] L. Silvestrini, Annu. Rev. Nucl. Part. Sci. 57, 405 (2007).

[101] J.A. Casas and S. Dimopoulos, Phys. Lett. B 387, 107 (1996).

[102] S. S. Adler et al. (E787 Collaboration), Phys. Rev. Lett. 88, 041803 (2002).

[103] S. S. Adler et al. (E787 Collaboration), Phys. Rev. D 70, 037102 (2004).

[104] C. Augusto et al., CERN-SPSC-2005-013; C. Biino and M. Pepe, arXiv:0809.4969.

[105] B. Aubert et al. (BABAR Collaboration), Phys. Rev. Lett. 94, 101801 (2005).

[106] A. Abulencia et al. (CDF Collaboration), Phys. Rev. Lett. 97, 242003 (2006).

[107] J. Foster, K.i. Okumura, and L. Roszkowski, J. High Energy Phys. 03 (2006) 044.

[108] A. G. Akeroyd et al. (SuperKEKB Physics Working Group), arXiv:hep-ex/0406071; M. Bona et al., arXiv:0709.0451; T.E. Browder, T. Gershon, D. Pirjol, A. Soni, and J. Zupan, arXiv:0802.3201 [Rev. Mod. Phys. (to be published)].

[109] A. Dedes, H. K. Dreiner, and P. Richardson, Phys. Rev. D 65, 015001 (2001).

[110] J. Alwall et al., J. High Energy Phys. 09 (2007) 028.

[111] V. M. Abazov et al. (D0 Collaboration), Phys. Rev. Lett. 90, 251802 (2003).

[112] T. Aaltonen et al. (CDF Collaboration), Phys. Rev. Lett. 101, 181602 (2008).

[113] L. Vacavant and I. Hinchliffe, J. Phys. G 27, 1839 (2001).

[114] J. Weng, G. Quast, C. Saout, A. De Roeck, and M. Spiropulu, CERN-CMS-NOTE-2006-129, 2006.

[115] J. H. Jeon, C. S. Kim, J. Lee, and C. Yu, Phys. Lett. B 636, 270 (2006).

[116] G. Buchalla, G. Hiller, and G. Isidori, Phys. Rev. D 63, 014015 (2000).

[117] W. Altmannshofer, A. J. Buras, D. M. Straub, and M. Wick, J. High Energy Phys. 04 (2009) 022. 